San Jose State University

SJSU ScholarWorks

Master's Theses

Master's Theses and Graduate Research

Spring 2012

\title{
Differential and Temporal Immunomodulation of alpha4 Integrins on CD4+ Memory Cells by Bordetella pertussis and Bordetella parapertussis
}

Dipti Ravindra

San Jose State University

Follow this and additional works at: https://scholarworks.sjsu.edu/etd_theses

\section{Recommended Citation}

Ravindra, Dipti, "Differential and Temporal Immunomodulation of alpha4 Integrins on CD4+ Memory Cells by Bordetella pertussis and Bordetella parapertussis" (2012). Master's Theses. 4165.

DOI: https://doi.org/10.31979/etd.ffqy-u4yk

https://scholarworks.sjsu.edu/etd_theses/4165

This Thesis is brought to you for free and open access by the Master's Theses and Graduate Research at SJSU ScholarWorks. It has been accepted for inclusion in Master's Theses by an authorized administrator of SJSU ScholarWorks. For more information, please contact scholarworks@sjsu.edu. 


\title{
DIFFERENTIAL AND TEMPORAL IMMUNOMODULATION OF ALPHA4 INTEGRINS ON CD4 ${ }^{+}$MEMORY CELLS BY BORDETELLA PERTUSSIS AND BORDETELLA PARAPERTUSSIS
}

\author{
A Thesis \\ Presented to \\ The Faculty of the Department of Biological Sciences \\ San José State University
}

In Partial Fulfillment

of the Requirements for the Degree

Master of Science

by

Dipti Ravindra

May 2012 
(C) 2012

Dipti Ravindra

ALL RIGHTS RESERVED 
The Designated Thesis Committee Approves the Thesis Titled

DIFFERENTIAL AND TEMPORAL IMMUNOMODULATION OF ALPHA4 INTEGRINS ON CD4 ${ }^{+}$MEMORY CELLS BY BORDETELLA PERTUSSIS AND BORDETELLA PARAPERTUSSIS

by

Dipti Ravindra

APPROVED FOR THE DEPARTMENT OF BIOLOGICAL SCIENCES

SAN JOSÉ STATE UNIVERSITY

May 2012

Dr. Tzvia Abramson - chair

Department of Biological Sciences

Dr. John Boothby

Department of Biological Sciences

Dr. Kenneth Youngman

Department of Biological Sciences 


\section{ABSTRACT \\ DIFFERENTIAL AND TEMPORAL IMMUNOMODULATION OF ALPHA4 INTEGRINS ON CD4 ${ }^{+}$MEMORY CELLS BY BORDETELLA PERTUSSIS AND BORDETELLA PARAPERTUSSIS}

by Dipti Ravindra

Pertussis, caused by Bordetella pertussis (B. pertussis), is reemerging worldwide due to vaccine inefficacy. The hallmarks of infection are extreme lymphocytosis and delayed recovery, which are partially associated with pertussis toxin. Lymphocytes migrate to infected tissues using trafficking receptors. Specific combinations of these lymphocyte trafficking receptors are identified for skin and gut but are not well established for lung.

This study focused on the effect of pertussis toxin on lung-associated trafficking receptors and tested the hypothesis that pertussis toxin alters dendritic cell imprinting of lung trafficking receptors on $\mathrm{T}$ cells, thus delaying resolution of the infection.

B. pertussis-infected mice were compared with pertussis toxin-deficient strains.

Imprinting of trafficking receptors on allogeneic $\mathrm{T}$ cells by dendritic cells derived from Bordetella-infected mice was analyzed by flow cytometry.

Mice infected with Bordetella strains showed an increase in mature dendritic cells on day 5 post-infection. Despite their mature phenotype, dendritic cells from $B$. pertussis infection, were compromised in their ability to imprint lung trafficking receptors on allogenic $\mathrm{T}$ cells. These results indicated a pertussis toxin-dependent defect in dendritic cell imprinting of lung trafficking receptors on $\mathrm{T}$ cells. In conclusion, this study provides important data for future vaccine development against respiratory pathogens. 


\section{ACKNOWLEDGEMENTS}

I would like to start by thanking Dr. Tzvia Abramson for accepting me as her Master's student and getting me interested in research. I never thought I would enjoy working in a lab, but thanks to her guidance and encouragement, I discovered how much I enjoyed research and what I was capable of accomplishing both in and out of the lab. I also want to thank Dr. John Boothby and Dr. Ken Youngman for their advice regarding my research project as well as reviewing and commenting on the manuscript. Additionally, I extend my gratitude to the staff at San José State University, including Tim Andriese, Art Valencia, Marco Parent, and the veterinary staff, Larry Young and Nelia Medeiros, for their help and support.

This research project was the combined efforts of several people. I could not have done it without the constant help of Tuan M. Nguyen and Ryan Ferguson throughout the course of this project. Also I would like to thank Sana Waheed and Brian Kwong for all their help and advice as well as all the other students who helped with the experiments.

Finally I wish to thank my husband, Uday Chandrasekhar and my parents Dr. Ravindra Kulkarni and Dr. Lakshmi Kulkarni without whose support and encouragement I couldn't have made it this far. 
TABLE OF CONTENTS

LIST OF FIGURES .................................................................................... vii

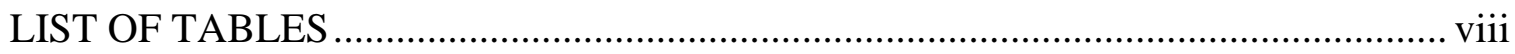

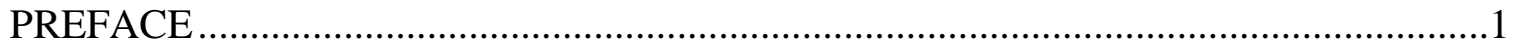

CHAPTER I

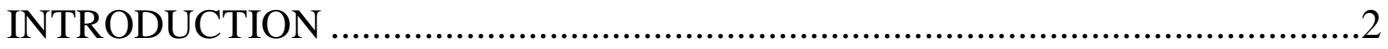

CHAPTER II

DIFFERENTIAL AND TEMPORAL IMMUNOMODULATION OF ALPHA4 INTEGRINS ON CD4 ${ }^{+}$MEMORY CELLS BY BORDETELLA PERTUSSIS AND BORDETELLA PARAPERTUSSIS.

CHAPTER III

CONCLUSION.

APPENDIX

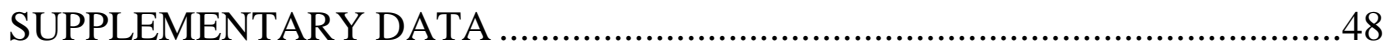

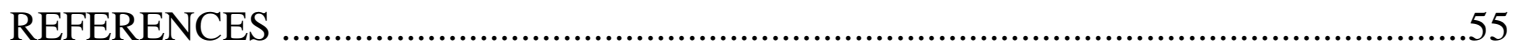




\section{LIST OF FIGURES}

FIG. 1. Leukocyte recruitment to the lungs of mice infected with Bordetella strains. ...26

FIG. 2. Trafficking receptor expression on emTh cells in blood and lungs from

B. pertussis- and B. parapertussis-infected mice at 5 and 25 days p.i.

FIG. 3. Functional effects of $\alpha 4$ integrins on migration and adhesion of blood

leukocytes from B. pertussis- and B. parapertussis-infection in vitro.

FIG. 4. Characterization of lung CD11 ${ }^{+}$cells during infection with $B$. pertussis and

B. parapertussis.

FIG. 5. $\alpha 4 \beta 7$ and $\alpha 4 \beta 1$ imprinting on allogenic Thy $1.1^{+} / \mathrm{CD} 4^{+} / \mathrm{CD} 38^{++}$cells.

FIG. A.1. B. pertussis induces a longer course of infection than B. parapertussis or

Bp $\triangle P T X$.

FIG. A.2. Blood immunophenotyping during the course of Bordetella infections.

FIG. A.3. Comparative trafficking receptor (TR) expression on emTh cells between

B. pertussis and $B p \triangle P T X$ infections.

FIG. A.4. Drastic increase of $\mathrm{CD} 11 \mathrm{c}^{+}$cells during the early phase of infection with

Bordetella strains.

FIG. A.5. Characterization of lung $\mathrm{CD} 11 \mathrm{c}^{+}$cells during infection with $B$. pertussis

and $B p \triangle P T X$.

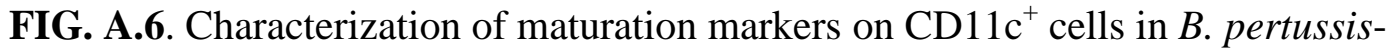

and $B$. parapertussis-infected mice at 25 days p.i.

FIG. A.7. Proliferation of the Thy $1.1^{+} / \mathrm{CD} 4^{+}$cells in allogenic system. 


\section{LIST OF TABLES}

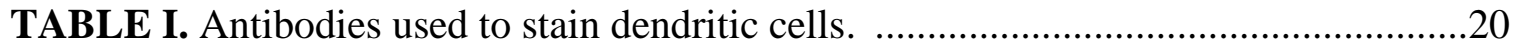

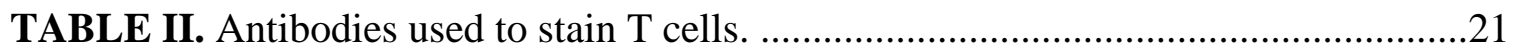




\section{PREFACE}

This thesis is comprised of three chapters and an appendix. Chapter I provides an overview of the research project, detailing the rationale behind the project design. Chapter II is presented in a journal format according to the guidelines set by the American Society for Microbiology's "Infection and Immunity.” Chapter III summarizes the conclusions of this work as well as discusses the future directions for this project. The appendix provides supplementary data associated with this study. 


\section{CHAPTER I}

\section{INTRODUCTION}




\section{OVERVIEW OF RESEARCH PROJECT}

Introduction/Background: Despite widespread vaccination of infants against

Bordetella pertussis (B. pertussis), the respiratory disease whooping cough is reemerging with high rates of morbidity and mortality worldwide. In the US, numerous states have declared a pertussis epidemic (40).

B. pertussis is a highly contagious pathogen that colonizes the respiratory tract by adhering to the bronchial ciliated epithelial cells. The hallmarks of this disease are a forceful "whooping" paroxysmal cough, a prolonged course of disease with recovery for up to several months, and extremely high number of blood-circulating lymphocytes (lymphocytosis). B. pertussis possesses a variety of very potent virulence factors that together concert its unique pathogenesis (39). Specifically, pertussis toxin (PTX) is linked to extreme lymphocytosis and the pathology associated with delayed recovery (43).

The immune system consists of a variety of lymphocytes that survey tissues and organs in the body for the appearance of foreign antigens. Their ability to enter the organs and identify pathogens is due to a crucial interaction of antigen-loaded, mature dendritic cells (DCs) and naïve lymphocytes in lymphoid organs. During this interaction, naïve lymphocytes are "educated" to become effector lymphocytes that respond to specific pathogens and upregulate trafficking receptors (TRs). This process of educating lymphocytes is called imprinting. It directs these cells to the area of infection where the antigen was originally picked up by the DCs (53). 
Successful antigen-mediated lymphocyte education is dependent on the recirculation of effector lymphocytes between the blood, lymphoid organs, and peripheral tissue (63), (64). The recirculation of lymphocytes is tightly controlled by the sequential association of adhesion molecules on endothelial cells of blood vessels with TRs on the lymphocytes. This process is termed lymphocyte trafficking or homing and is responsible for the recruitment of $\mathrm{T}$ cells to sites of infection (14). Unique combinations of TRs direct lymphocytes to target organs (14). Many of these combinations are well described for cells migrating to the gut ( $\alpha 4 \beta 7$, CCR9 CCR10) and the skin (CCR4 and CCR10). The combination of TRs for specifying respiratory homing has not been uncovered (18). However, TR combinations that are important for homing of lymphocyte to other mucosal tissue, such as $\alpha 4 \beta 7$ and $\alpha 4 \beta 1$, are also involved in lymphocyte migration to mucosal airways (51).

Hypothesis: In order to understand the prolonged resolution of pertussis infection, this study tested the hypothesis that during B. pertussis infection, PTX alters the imprinting of mucosal- and lung-associated TRs on T cells leading to compromised T cell migration to the lung, and thus to prolonged recovery from the disease.

Approach and Methodology: To verify the unique effect of PTX, we used two models of natural human respiratory infections: B. pertussis, a human respiratory pathogen that expresses PTX, and Bordetella parapertussis (B. parapertussis), a related human pathogen that does not express PTX (6), but overlaps with $90 \%$ of the B. pertussis genome. We included a PTX-deleted B. pertussis strain Bordetella pertussis- $\triangle P T X$ $(B p \triangle P T X)$ to further confirm the effect of PTX on imprinting of mucosal- and lung- 
associated TRs on T lymphocytes. The experiments were conducted during two phases of infection. The early phase occurred 5 days post-infection (p.i.), when maximum numbers of bacteria were isolated from the lungs and blood leukocytes peak in all three infectious models. Late phase occurred 25 days p.i., when no bacteria were isolated from the lungs and blood leukocyte numbers dropped in all infection models.

It has been well documented that PTX inhibits Gai that mediates signaling of Gprotein-coupled receptor (GPCR) and chemoattractant responses (61). Some of the GPCR are essential for mature DCs to migrate into lymphoid organs where imprinting of TRs on T lymphocytes occurs (58). Thus, we proposed the following two aims for this study.

The first aim was to determine the trafficking patterns of leukocytes from mice infected with Bordetella species. To study TR patterns, we performed the following experiments: A) Hemoatoxylin and eosin ( $\mathrm{H} \& \mathrm{E})$ and immunofluorescence staining of lung sections to detect leukocyte recruitment to infected lungs; B) Flow cytometry to characterize TRs on effector T cells in blood and lung; C) In vitro leukocyte migration studies to establish the functional role of $\alpha 4$ integrin.

The second aim of this project was to determine the effect of $B$. pertussis and PTX on TR imprinting on effector T cells. Since origin and phenotype of DCs is vital for TR imprinting on T lymphocytes in lymphoid organs, lung DCs from Bordetella-infected mice at 5 and 25 days p.i. were phenotyped by flow cytometry. Additionally, isolated lung DCs from all infection models at 5 days p.i. were combined with naïve allogeneic T 
cells in vitro. The TRs imprinted on allogenic T cells during these interactions were analyzed by flow cytometry in all infection models.

Chapter II, presented in a journal format (American Society of Microbiology's "Infection and Immunity"), includes results from both aims, but the thesis focuses mainly on the second aim of the overall study, since these experiments were conducted exclusively by Dipti Ravindra for MS thesis.

Results and Discussion: To determine that a respiratory immune response was initiated following the infection with Bordetella species we analyzed lung sections for general cell recruitment by hematoxylin and eosin staining and phenotyped the cells by immunofluorescence staining.

Hematoxylin and Eosin, and Immunofluorescence staining: At 5 days p.i. lungs from $B$. parapertussis- and $B p \triangle P T X$-infected mice were infiltrated mainly by neutrophils. No cell accumulation was observed in lungs of $B$. pertussis-infected mice. This manifestation was reversed at 25 days p.i. when airways reverted to uninfected conditions in B. parapertussis- and Bp $\triangle P T X$-infected mice. In lungs from B. pertussis-infected mice, immunofluorescence staining displayed a combined population of leukocytes consisting of T cells and B cells surrounding the airway, and neutrophils infiltrating the lung parenchyma. These results indicate a delay in recruitment of immune cells to the lungs of mice infected with $B$. pertussis, thus confirming the postponed recovery in this infection.

Dendritic cell phenotyping: Since the origin and phenotype of DC is crucial for TR imprinting (56), we analyzed lung DCs in the various experimental groups. 
Compared to uninfected mice, the proportions of DCs that expressed the maturation markers, MHC class II and CD86, at 5 days p.i. were 3.28-fold and 1.77-fold higher in B. pertussis-infected mice, and 3.28-fold and 1.75-fold higher in B. parapertussisinfected mice, respectively suggesting that both natural infections are equally and immediately recognized by the immune system. At 25 days p.i., the proportion of MHC class II and CD86 expressing DCs resolved to uninfected levels in B. parapertussis- and $B p \triangle P T X$-infected mice but continued to be high in $B$. pertussis-infected mice, which was 4.87-fold and 3.36-fold above similar cells from uninfected mice, respectively. These data indicate prolonged exposure of lung DCs to B. pertussis-antigens, which additionally confirms the prolonged nature of this infection. Expression of CCR7 (a GPCR) is necessary for mature DCs to exit tissue and enter the lymphatics to participate in the imprinting process in the draining lymph nodes (58). The proportion of lung DCs that expresses CCR7 in B. pertussis infection was significantly reduced compared to those from B. parapertussis infection, which was 0.64-fold and 0.91-fold, respectively, compared to similar cells from uninfected mice at 5 days p.i. The reduced expression of CCR7 may suggest a diminished ability of lung DCs from B. pertussis-infected mice to exit the respiratory tissue and reach the lymphoid organs.

Trafficking receptor in co-culture systems: TR imprinting capabilities of lungderived DCs from 5 days p.i. were verified in an allogenic system. Cell proliferation of allogenic T cells was confirmed by 5(6)-carboxyfluorescein succinimidyl ester (CFSE) proliferation assay. Four generations of cell-cycle proliferation were observed in each of the three Bordetella infection models, suggesting lung-derived DCs from all systems 
proportionately induced proliferation of allogenic $\mathrm{T}$ cells. Despite $\mathrm{T}$ cell proliferation in all three systems, the proportion of allogeneic T cells that expressed $\alpha 4 \beta 7$ and $\alpha 4 \beta 1$ in B. pertussis infection were 0.77 -fold and 0.46 -fold, 1.32 -fold and 2.32-fold for cells from B. parapertussis-infected mice and 1.14-fold and 1.03-fold for cells from Bp $\triangle P T X$ infected group compared to cells from uninfected mice at 5 days p.i. These results indicate that $\alpha 4 \beta 1$ was significantly reduced in the system comprising lung DCs from B. pertussis infection compared to cells co-cultured with lung DCs from B. parapertussis or $B p \triangle P T X$-infected mice. In this study $\alpha 4 \beta 7$ imprinting did not statistically vary between all allogeneic systems, suggesting that additional mechanisms may contribute to PTX-dependent $\alpha 4 \beta 7$ expression on T cells.

Conclusion and Significance: Our results suggest that during B. pertussis infection although PTX does not alter DC maturation, the percentage of T cells expressing mucosal- and lung-associated TRs is reduced, leading to prolonged recovery from the disease. Therefore, vaccines directed at $B$. pertussis should address the expression of mucosal- and lung-associated TRs on T cells for an effective cellularmediated immune response. 
CHAPTER II

DIFFERENTIAL AND TEMPORAL IMMUNOMODULATION OF ALPHA4 INTEGRINS ON CD4 $4^{+}$MEMORY CELLS BY BORDETELLA PERTUSSIS AND BORDETELLA PARAPERTUSSIS 


\title{
DIFFERENTIAL AND TEMPORAL IMMUNOMODULATION OF ALPHA4 INTEGRINS ON CD4 ${ }^{+}$MEMORY CELLS BY BORDETELLA PERTUSSIS AND BORDETELLA PARAPERTUSSIS
}

\author{
Dipti Ravindra ${ }^{1}$, Tuan M. Nguyen ${ }^{1}$, Brian Kwong ${ }^{1}$, Sana Waheed ${ }^{1}$, Ryan Ferguson ${ }^{1}$, \\ Nicole Tarlton ${ }^{1}$, Martina Bremer ${ }^{2}$, and Tzvia Abramson* ${ }^{1}$
}

${ }^{1}$ San José State University, Department of Biology, One Washington Square, San José, CA 95192; ${ }^{2}$ San José State University, Department of Mathematics, One Washington Square, San José, CA 95192

Key words: Whooping cough, Bordetella pertussis, pertussis toxin, integrin receptor, lymphocyte homing

Correspondent Footnote: Tzvia Abramson, Department of Biological Sciences, One Washington Square, San José, CA 95192

(Phone: 408-924-4872, Fax: 408-924-4840. E-mail: tzvia.abramson@sjsu.edu) 


\section{ABSTRACT}

Pertussis, a respiratory infection caused by the bacterium Bordetella pertussis $(B p)$, is reemerging due to decreased vaccine efficacy. Mechanisms of selective trafficking of lymphocytes to airways are likely to be critical in the immune response against this airborne pathogen. Here, we study pertussis toxin (PTX)-dependent mucosal- and lung-associated trafficking receptor (TR) expression and imprinting on effector/memory T helper (emTh) cells in a mouse model of Bordetella infection.

Increased cell recruitment to the lungs, at 5 days post infection (p.i.) with Bordetella parapertussis (Bpp) and B. pertussis- $\triangle P T X(B p \triangle P T X)$ coincides with an increased frequency of $\alpha 4 \beta 7$ and $\alpha 4 \beta 1$ expression in circulating emTh cells. While these TRs are reduced in the $B p$ mouse model at 5 days p.i., they are highly evident at 25 days p.i., when $B p p$ and $B p \triangle P T X$ infections are resolved. Although a lower percentage of $\mathrm{Bp}$-lung dendritic cells (DCs) express CCR7 at 5 days p.i., compared to Bpp-lung DCs, an equally high percentage of these cells express MHC class II and CD86, these markers persist up to 25 days p.i. exclusively in $B p$-lung DCs. In addition, a reduced frequency of allogeneic $\mathrm{CD}^{+}$cells expressing only $\alpha 4 \beta 1$ is detected following the co-culture with Bp-lung DCs.

Our findings suggest that during infection with $B p$, but not with $B p p$ or $B p \triangle P T X$, a reduced number of emTh cells display mucosal- and lung-associated TRs due to a PTXdependent defect on DC imprinting capabilities. This explains delay in resolution of B. pertussis infection and implies that modulators of immune cell trafficking should be considered in the design of new vaccines. 


\section{INTRODUCTION}

Despite widespread vaccination against Bordetella pertussis (B. pertussis) in infants, whooping cough is reemerging with high rates of morbidity and mortality worldwide. In the U.S., several states have declared pertussis epidemics (40). Due to waning efficacy of the acellular vaccine, adults may acquire whooping cough despite vaccination in infancy. Many of those afflicted will experience only a mild disease that may pass undetected and serve as a reservoir of infection for unimmunized infants, who are then at risk for developing a profound and deadly respiratory disease. Thus, whooping cough continues to be a worldwide problem and a better understanding of B. pertussis pathogenesis may lead to a more effective vaccine.

B. pertussis is a highly contagious human pathogen that colonizes the respiratory tract by adhering to ciliated bronchial epithelial cells. Hallmarks of the disease are a forceful "whooping" paroxysmal cough, extreme lymphocytosis, and a prolonged course of disease with recovery for up to several months. A variety of potent virulence factors work in concert to contribute to the unique pathogenesis of B. pertussis (39).

Specifically, pertussis toxin (PTX) is linked to the extreme lymphocytosis and the pathology associated with delayed recovery (43).

PTX, an AB-subunit toxin, is both bacterial surface-associated and secreted as a soluble toxin. Recently it was reported that influenza virus infection and $B$. parapertussis infection are enhanced by $B$. pertussis during a mixed infection of respiratory tract likely due to the suppressive effect of PTX on innate immunity during early stages of infection (9), (65). The catalytic A-subunit (PTX-A) is well documented to inhibit G-protein- 
coupled receptors (GPCRs) and chemoattractant responses (61). During B. pertussis infection, this function leads to the intoxication of several cell types in the respiratory tissues, such as airway macrophages (3), bronchial epithelial cells, and pulmonary endothelial cells (45). This detrimental effect is thought to delay neutrophil recruitment to the lungs during early phase of infection $(3,4)$.

The B-subunit (PTX-B) displays a more diverse role; it is documented to facilitate B. pertussis colonization and the binding of soluble PTX to a large variety of glycosylated cellular receptors (16). In addition, PTX-B is extremely immunogenic (44) and thus widely used in the pertussis acellular vaccines. Late-induced Th1/Th17 immune responses are linked to PTX-B function and perhaps to the eventual resolution of B. pertussis infection and disease (5). Nevertheless, in vitro studies demonstrate that PTX-B is responsible for the neutralization of inflammatory receptors and responses (35), (4), (21) as well as desensitization of the chemokine receptor CXCR4 in Jurkat cells (54).

Despite ample documentation of PTX function, the effects of B. pertussis and PTX on adaptive immune responses, specifically on lymphocyte trafficking receptors (TRs) responsible for homing to the airways is not well understood. Peripheral blood lymphocytes from infants infected with $B$. pertussis show reduced expression of CD62L, an adhesion receptor facilitating lymphocyte-endothelial cell interactions (30), (28). This implies that $B$. pertussis may also alter lymphocyte trafficking by mechanisms that do not directly involve G-protein blocking.

It is well established that $\mathrm{T}$ and B lymphocytes circulate among lymphoid tissues and the peripheral organs to identify new invading pathogens, to develop and sustain an 
immune defense, and to resolve infection (14). The recirculation of lymphocytes is tightly controlled by the sequential association of adhesion molecules with chemoattractant receptors [generally termed "trafficking receptors" (TRs)]. Expression of these receptors is "imprinted" in lymphoid tissues during the interaction of specialized dendritic cells (DCs) with naïve T cells in a specific cytokine environment (14). Unique combinations of TRs direct lymphocytes to tissues in a selective fashion and these combinations are well documented for lymphocyte trafficking to the gut and skin (66), (48), (47). However, no unique TR combinations are identified for respiratory homing (19). Still, T lymphocytes recirculating through the lungs display lower levels of $\alpha 4 \beta 7$ than their intestinal counterparts, and lack CCR9 (66). These cells do not express skinassociated CCR10 or high levels of cutaneous lymphocyte antigen (CLA), and they express lower levels of CCR4 than skin T cells (48), (47). Instead, integrin receptor $\alpha 4 \beta 1$ (VLA-4), collagen-binding very late antigen-1 (VLA-1 or $\alpha 1 \beta 1)(51)$, leukocyte function associated antigen-1 (CD11a or LFA-1) (60), and perhaps CD49f $(\alpha 6)$ and P-selectin ligand (P-sel lig) (17) are suggested to participate in T cell homing to the lungs (1), (48), (47), (15).

In this study we hypothesized that despite the extreme lymphocytosis observed during infection with $B$. pertussis, circulating lymphocytes display an altered pattern of TR expression due to a PTX-dependent imprinting defect, leading to a prolonged disease and a delayed resolution of the infection. We tested this hypothesis using two organisms responsible for human respiratory infections. B. pertussis, a human respiratory pathogen that expresses PTX, and Bordetella parapertussis (B. parapertussis), a related human 
pathogen that does not express PTX (7), but whose genome overlaps with $90 \%$ of B. pertussis. We further investigated PTX-dependent effects using the PTX-deleted B. pertussis strain, B. pertussis- $\triangle P T X(B p \triangle P T X)$.

We show that while $B$. parapertussis infection immediately induces a high percentage of circulating effector/memory T helper (emTh) cells that express $\alpha 4 \beta 7$ and $\alpha 4 \beta 1$, this response is delayed for several weeks in B. pertussis infection and results in inhibited leukocyte migration. Furthermore, while lung DCs derived from mice infected with $B$. pertussis display a persistent maturation status, they demonstrate a reduced capacity to imprint $\alpha 4 \beta 1$ on allogeneic $\mathrm{CD} 4^{+}$cells following their exclusive co-culture with $B$. pertussis lung DCs. We suggest that $\alpha 4$ integrins, in combination with $\beta 7$ and $\beta 1$, play an important role in emTh cell migration during B. pertussis infection, and that the disruption of TR imprinting associated with PTX may explain the delay in disease resolution caused by this pathogen. 


\section{MATERIALS AND METHODS}

\section{Mice}

All studies using animals were approved by the Institutional Animal Care and Use Committee (IACUC) at San José State University, San José, CA (protocol \# 921), and all animals were handled in accordance with institutional guidelines. Female BALB/c mice and female FVB/N (Thy $1.1^{+}$) mice were purchased from Simonsen Labs (Gilroy, CA).

\section{Bacteria}

Three Bordetella strains were used: B. pertussis 338, which is a nalidixic acidresistant derivative of Tohama I (62), B. parapertussis (ATCC 9305), and mutant B. pertussis- $\triangle P T X$, which contains a deletion of the 3.4-kb BstEll fragment encompassing the PTX operon. Bordetella strains were grown on Bordet-Gengou blood agar plates. A single colony from the agar plates was used to inoculate Stainer-Scholte broth supplemented with heptakis (2, 6-O-dimethyl beta-cyclodextrin) (Sigma-Aldrich, St. Louis, MO), casamino acid, and proline three days prior to experimental infection. The liquid cultures were grown at $37^{\circ} \mathrm{C}$ on an orbital shaker $(180 \mathrm{rpm})$, and cultures were split every day for three days to maintain a log phase of growth before confirming cell concentration at optical density (OD) of $600\left[1 \mathrm{OD}=3 \times 10^{9}\right.$ colony forming units $(\mathrm{CFU}) / \mathrm{ml}]$. Cells were pelleted and resuspended in PBS at a concentration of $5 \times 10^{6}$ $\mathrm{CFU} / 20 \mu \mathrm{l} \mathrm{PBS}$. 


\section{Infection}

Six-week-old female BALB/c mice were infected intranasally (i.n.) with $20 \mu \mathrm{l}$ $5 \times 10^{6} \mathrm{CFU}$ of B. pertussis, B. parapertussis, or Bp $\triangle P T X$. Uninfected mice (mock) received $20 \mu \mathrm{l}$ PBS (12). Two time points of experimental infection were established, 5 days post-infection (p.i.) (early stage) and 25 days p.i. (late stage). These time points were previously determined through a time-course experiment in which bacterial load in the lungs was measured consecutively for 10 days and again at 25 days p.i. (Fig. A1, see Appendix). In brief, lungs from experimentally infected mice were harvested, homogenized, and plated on Bordet-Gengou blood agar plates in order to confirm colonization of the lungs and assess the bacterial load.

\section{Preparation of blood leukocytes: dextran sedimentation}

Leukocyte suspensions were prepared by dextran sedimentation as previously described (25). In brief, whole blood was obtained by cardiac puncture and mixed with a solution containing $1.5 \mathrm{mM}$ EDTA and $2 \%$ dextran, and incubated at $37^{\circ} \mathrm{C}$ for 30 minutes. Leukocyte-rich plasma, located on the top of the aggregated erythrocytes, was carefully collected and centrifuged at 300xg for 5 minutes. The cell pellets were mixed with $3 \mathrm{ml}$ red blood cell lysis buffer (Sigma-Aldrich, St. Louis, MO) for 5 minutes and washed with RPMI-1640 containing 10\% fetal calf serum (FCS ). Cells were stained for flow cytometry analysis (BD LSRII, Stanford University, Palo Alto, CA) or used for in vitro migration and adhesion assays. 


\section{Preparation of lung mononuclear cells}

Lungs from infected BALB/c mice were perfused with 30-50 ml PBS. The lungs and trachea were removed, minced, and then digested in RPMI-1640 media containing 10\% FCS, 75 U/ml DNase I (Sigma-Aldrich, St. Louis, MO), and 250 U/ml Type I collagenase (Worthington Biochemical Corporation, Lakewood, $\mathrm{NJ}$ ) in a $37^{\circ} \mathrm{C}$ orbital shaker for 45-60 minutes ( $5 \mathrm{~mL}$ media/lung). The digested lungs were passed through a $70 \mu \mathrm{m}$ nylon mesh strainer, and cells were washed with RPMI-1640 to remove residual digest medium. Digested lung tissue was resuspended in 40\% Percoll (GE Lifescience, Piscataway, NJ), slowly under-laid with 70\% Percoll, and centrifuged at 300xg for 25 minutes (without brake) to create a density gradient. Cells at the $40-70 \%$ interface (mononuclear cells) were collected and stained for flow cytometry analysis or further used for enrichment of DCs.

\section{Enrichment of lung dendritic cells}

Lung mononuclear cells, obtained via percoll density gradient, were enriched for DCs $\left(\mathrm{CD} 11 \mathrm{c}^{+}\right)$by positive selection using directly conjugated anti-CD11c microbeads (N418) with a MiniMACS ${ }^{\mathrm{TM}}$ separator (Miltenyi Biotech, Auburn, CA). In brief, lung mononuclear cells were incubated with $100 \mu \mathrm{l} \mathrm{CD11c} \mathrm{microbeads} \mathrm{for} 15$ minutes at $4^{\circ} \mathrm{C}$. Cells were then washed with MACS buffer (PBS, 0.5\% FCS, 2 mM EDTA) and resuspended in $500 \mu 1$ of the same buffer. Cells were then loaded on one MACS $^{\circledR}$ MS column (Miltenyi Biotech, Auburn, CA), which enriched for CD11 ${ }^{+}$cells. Then the cells were either stained for flow cytometry analysis (BD LSRII, Stanford University, Palo Alto, CA) or used in co-culture experiments. 


\section{Splenocyte preparation}

The spleens of six-week-old female FVB/N Thy $1.1^{+}$mice were harvested and pressed through a nylon mesh to obtain a single cell suspension (8). Splenocytes were incubated with $3 \mathrm{ml}$ RBC lysis buffer (Sigma-Aldrich, St. Louis, MO) for 5 minutes and washed with RPMI-1640 containing 10\% FCS. Cell pellets from the spleens were resuspended in PBS buffer containing $0.5 \%$ bovine serum albumin (BSA) and $2 \mathrm{mM}$ EDTA.

\section{Enrichment of allogeneic $\mathrm{CD4}^{+} \mathrm{T}$ cells}

Splenocytes obtained from female FVB/N Thy1.1 mice were enriched for CD4 ${ }^{+}$ T cells by positive selection using directly conjugated anti-CD4 microbeads (L3T4) with a MidiMACS $^{\mathrm{TM}}$ or QuadroMACS ${ }^{\mathrm{TM}}$ separator (Miltenyi Biotech, Auburn, CA). In brief, splenocytes were incubated with $200 \mu \mathrm{l} \mathrm{CD} 4$ microbeads for 15 minutes at $4^{\circ} \mathrm{C}$. Cells were then washed with MACS buffer (PBS, 0.5\% FCS, 2 mM EDTA) and resuspended in $500 \mu 1$ of the same buffer. Cells were then loaded on one MACS ${ }^{\circledR}$ LS column (Miltenyi Biotech, Auburn,CA), which was used to enrich for $\mathrm{CD}^{+}$cells.

\section{Co-culture experiments}

Lung DCs enriched by CD $11 c^{+}$positive selection were co-cultured in a $24-$ well flat-bottomed plate with positively selected $\mathrm{CD}^{+}$cells from naïve FVB/N Thy $1.1^{+}$ allogeneic mice (1:5 ratio) in $500 \mu \mathrm{l}$ RPMI-1640 media with $10 \%$ FCS, and $1 \%$ each of penicillin and streptomycin (RPMI complete media). Cells were co-cultured for 4 days at 
$37^{\circ} \mathrm{C}$ with $5 \% \mathrm{CO}_{2}$ to allow for $\mathrm{T}$ cell proliferation, and TR imprinting, followed by flow cytometry analysis.

\section{T cell proliferation experiments}

T cell proliferation was determined by 5(6)-carboxyfluorescein succinimidyl ester (CFSE) dye dilution. Briefly, positively selected CD4 ${ }^{+}$cells from allogeneic FVB/N Thy $1.1^{+}$mice were labeled with CFSE as previously described (37). Subsequently, they were co-cultured for 4 days with positively selected lung DCs $\left(\mathrm{CD} 11 \mathrm{c}^{+}\right)$in $500 \mu \mathrm{RPMI}$ complete media at $37^{\circ} \mathrm{C}$ with $5 \% \mathrm{CO}_{2}$, followed by flow cytometry analysis.

\section{Flow cytometry}

Table I. Antibodies used to stain dendritic cells.

\begin{tabular}{|l|l|l|}
\hline \multicolumn{1}{|c|}{ Marker } & \multicolumn{1}{|c|}{ Clone } & \multicolumn{1}{c|}{ Source } \\
\hline CD11c & N418 & BioLegend \\
CD11b & M1/70 & BioLegend \\
CD207 & eBioL31 & eBioscience \\
MHC- II & M5/114.15.2 & BioLegend \\
CD86 & GL-1 & BioLegend \\
CCR7 & $4 B 12$ & BioLegend \\
CD103 & $2 E 7$ & BioLegend \\
CXCR4 & $2 B 11$ & eBiosicence \\
\hline
\end{tabular}


Table II. Antibodies used to stain T cells.

\begin{tabular}{|c|c|c|c|c|c|}
\hline \multicolumn{3}{|c|}{ Memory/Activated T cells Markers } & \multicolumn{3}{|c|}{ Trafficking Receptors } \\
\hline Marker & Clone & Source & Marker & Clone & Source \\
\hline CD4 & RM4-5 & $\begin{array}{l}\text { BD } \\
\text { Bisociences }\end{array}$ & CD29 $(\beta 1)$ & HMb1-1 & BioLegend \\
\hline CD38 & 90 & BioLegend & CD49d $(\alpha 4)$ & $9 \mathrm{C} 10$ & BioLegend \\
\hline Thy1.1 & $\mathrm{OX}-7$ & eBioscience & $\beta 7$ & FIB27 & BioLegend \\
\hline CD44 & IM7 & BioLegend & CD11a & M17/4 & BioLegend \\
\hline CD45RB & C363-16A & BioLegend & CD162 & 2PH1 & BD Biosciences \\
\hline
\end{tabular}

Lung-derived DCs were stained with antibodies as indicated in Table I. T cells were stained with antibodies as indicated in Table II (BioLegend San Diego, CA; eBioscience San Diego, CA; BD Biosciences San Diego, CA). Stained cells were acquired on a BD Biosciences LSRII flow cytometer (Butcher Lab at Stanford University, Palo Alto, CA) and analyzed using FlowJo software (Tree Star Inc., Ashland, OR). The frequency of cells expressing the above trafficking markers were compared for B. pertussis and B. parapertussis infections and normalized to uninfected controls as explained in the figure legends.

\section{Hematoxylin and eosin staining of lung sections}

The lung of uninfected and infected BALB/c mice, inoculated i.n., were infused with a 1:1 ratio of PBS and Optimal Cutting Temperature (OCT) embedding media, and frozen in only OCT media on dry ice. The lungs were cut into $6 \mu \mathrm{m}$ sections and fixed with cold acetone. They were subsequently stained with hematoxylin and eosin (H\&E). Ten randomly selected fields (400x) from each lung were photographed. 


\section{Immunofluorescence of lung sections}

Macrophage and B cell staining: Acetone-fixed frozen lung sections were washed with 1 X TBS containing $0.1 \%$ Tween 20 and blocked with PBS containing 5\% goat serum. The sections were sequentially incubated with F4/80 rat anti-mouse monoclonal antibody (mAb) (CI:A3-1, 1:200 dilution for $1 \mathrm{hr}$ ) or control mAb, FITC goat anti-rat antibody (Jackson ImmunoResearch, Westgrove, PA; 1:500 dilution for $30 \mathrm{~min}$ ), PBS with 5\% rat serum (Lampire Laboratory, for 30 minutes), goat anti-rat IgG Fab fragment (Jackson ImmunoResearch, Westgrove, PA; 1:200 dilution for 30 minutes), CD45R/B220 rat anti-mouse mAb (RA3-6B2, 1:200 dilution for 1 hour) or control mAb, and Dylight 594 goat anti-rat antibody (Jackson ImmunoResearch, Westgrove, PA; 1:500 dilution for 30 minutes). Slides were counterstained with DAPI.

Neutrophil and T cell staining: Acetone-fixed frozen sections of lung were washed with $1 \mathrm{X}$ TBS containing $0.1 \%$ Tween 20 , and blocked with PBS containing 5\% goat serum. The sections were sequentially incubated with CD3 hamster anti-mouse mAb (145-2C11, 1:200 dilution for 1 hour) or control mAb, Dylight 488 goat antihamster antibody (Poly4055, 1:500 dilution for 30 minutes), Gr-1 rat anti-mouse mAb (RB6-8C5, 1:200 dilution for 1 hour) or control mAb, and Dylight 594 goat anti-rat antibody (Jackson ImmunoResearch, Westgrove, PA; 1:500 dilution for 30 minutes). Slides were counterstained with DAPI.

Sections were visualized at 100x magnification using a LEICA confocal microscope (DMI-4000B) at San José State University. All antibodies used for 
immunofluorescence staining were purchased from BioLegend (San Diego, CA) unless otherwise indicated.

\section{Competitive $\alpha 4$-dependent chemotaxis in vitro migration}

Chemotaxis was performed with transwell tissue culture plates $(3 \mu \mathrm{m}$ pore size) purchased from Corning Costar. The transwells were coated with mouse VCAM-1 (100 $\mu \mathrm{g} / \mathrm{ml}$; R\&D Systems, Minneapolis, MN) overnight at $4{ }^{\circ} \mathrm{C}$ and then blocked with $2 \%$ BSA for 30 minutes at room temperature. An aliquot of $0.5 \times 10^{5}$ blood leukocytes each from B. pertussis (CFSE labeled; Invitrogen Grand Island, NY) (37) and B. parapertussis (eFluor 670 labeled; eBioscience San Diego, CA) (55) were suspended in $100 \mu \mathrm{l}$ chemotaxis buffer (RPMI-1640, 0.5\% BSA) and loaded onto the upper chamber of the transwells. Stromal cell-derived factor-1a (SDF-1a, 100 ng/ml; Miltenyi Biotech, Auburn, CA) was added to the lower chamber. Neutralizing anti- $\alpha 4$ monoclonal antibody (5 $\mu \mathrm{g} / \mathrm{ml}$ PS/2,) (gift from Dr Butcher, Stanford University) was added to block the cells in the upper chamber where indicated in Fig. 3A. The cells were allowed to migrate for 4 hours at $37^{\circ} \mathrm{C}$, and the cells that migrated to the lower chamber were enumerated by flow cytometry (BD FACS Calibur; BD Biosciences, San José, CA).

\section{Cell adhesion assay}

A 96-well plate was coated with mouse vascular cell adhesion molecule-1 (VCAM-1) $(10 \mu \mathrm{g} / \mathrm{mL})$ overnight at $4^{\circ} \mathrm{C}$, and then blocked with $2 \%$ BSA for 30 minutes at room temperature. An aliquot of $0.5 \times 10^{5}$ blood leukocytes from B. pertussis or B. parapertussis treated mice was loaded into the wells. After 40 minutes of incubation 
at room temperature, the wells were washed three times. The adhering cells were stained with $0.2 \%$ crystal violet in $10 \%$ ethanol. The stain was solubilized using a 1:1 mixture of $0.1 \mathrm{M} \mathrm{NaH}_{2} \mathrm{PO}_{4}(\mathrm{pH} 4.5)$ and $50 \%$ ethanol, and absorbance was measured at $595 \mathrm{~nm}$ on a microplate reader. 


\section{RESULTS}

\section{Delayed recruitment of leukocytes to the lungs observed solely in B. pertussis infection}

While similar numbers of CFUs were retrieved at 5 days p.i. from the lungs of mice infected with either of the three Bordetella strains, the H\&E analyses showed a dense cell infiltration only in B. parapertussis lung sections, and to a lesser degree in $B p \triangle P T X$-infected lungs. No significant cell accumulation was observed in the lungs of B. pertussis infected mice at that time point (Fig. 1A). However, this occurrence was reversed at 25 days p.i. where no cell accumulation was demonstrated in B. parapertussis and Bp $\triangle P T X$ lungs, but a massive recruitment of cells was observed in the lungs of B. pertussis infected mice.

Similarly, the immunofluorescence staining of lung sections revealed a predominant infiltration of neutrophils at 5 days p.i. in $B$. parapertussis- and $B p \triangle P T X$ infected lungs, but no significant recruitment of any of these cells was detected in lungs derived from the $B$. pertussis-infected mice similar to lung sections from uninfected mice. Furthermore, at 25 days p.i., no significant accumulation of immune cells was observed in lung sections derived from B. parapertussis or Bp$\triangle P T X$ infections, while leukocyte accumulation was detected in lung sections from the B. pertussis infection, where neutrophils occupied the lung parenchyma, and T and B cells clustered mainly around airways in this model (Fig. 1B). 
1A. Uninfected B. pertussis B. parapertussi BpAPTX
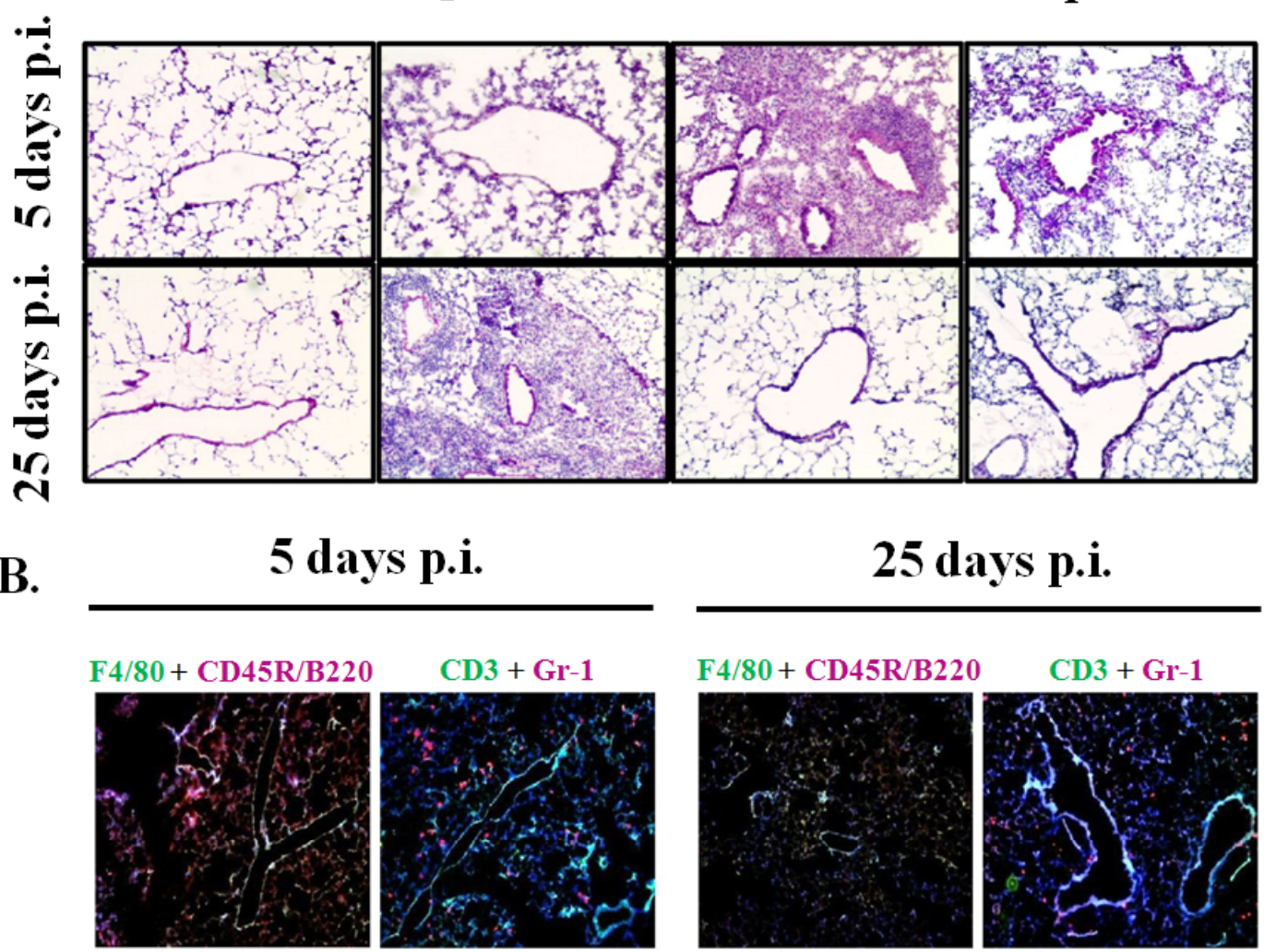

$\mathrm{CD} 3+\mathrm{Gr}-1$
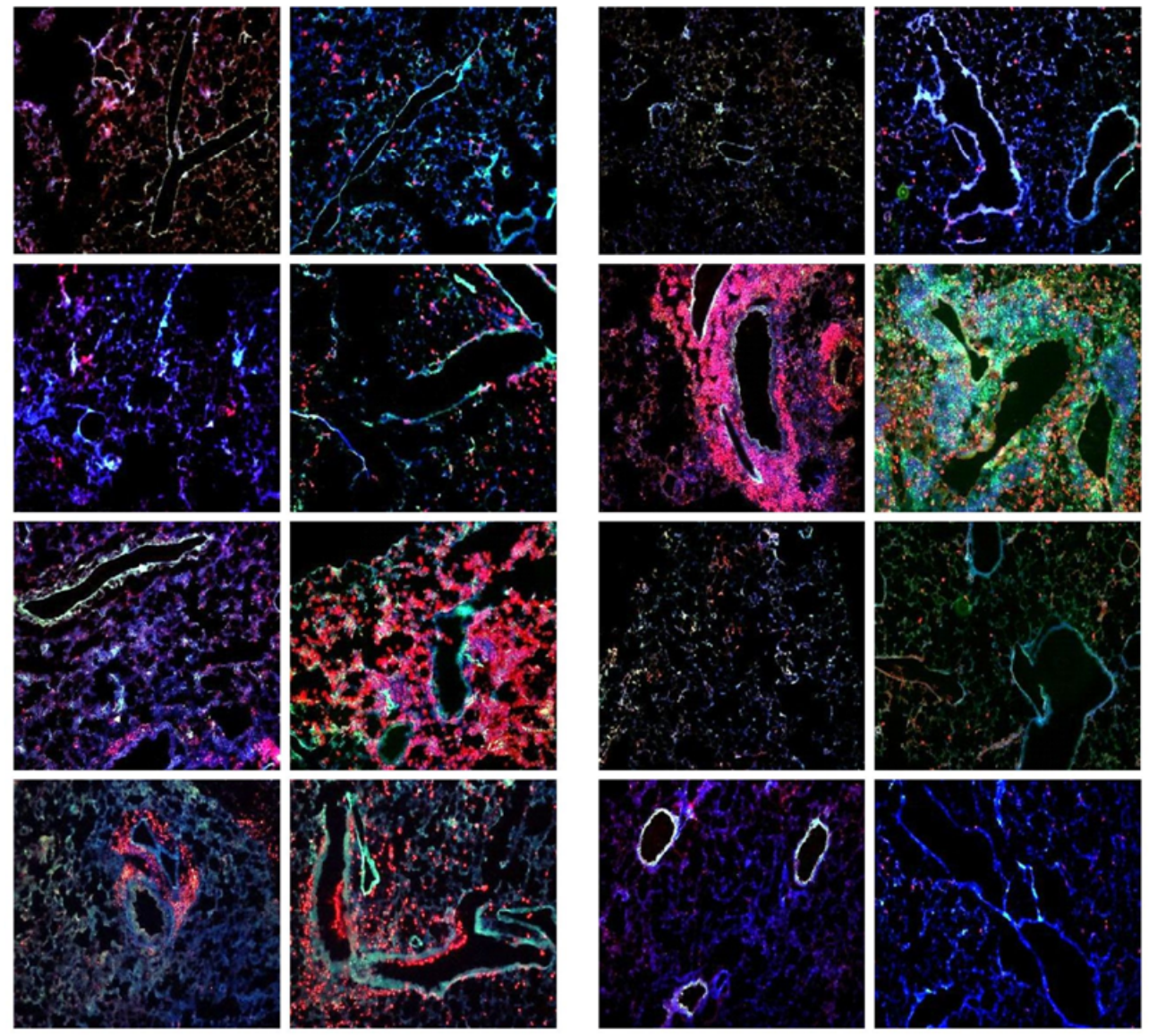
FIG. 1. Leukocyte recruitment to the lungs of mice infected with Bordetella strains. (A) Frozen sections of lungs from BALB/c mice infected with $B$. pertussis, B. parapertussis, Bp $\triangle P T X$ or uninfected at 5 and 25 days p.i were stained with hematoxylin and eosin. Ten independent fields from each infection were photographed under 400x magnification. (B) Frozen lung sections from BALB/c mice infected with $B$. pertussis, $B$. parapertussis, Bp $\triangle P T X$, or uninfected at 5 and 25 days p.i. were stained sequentially with F4/80 (green) for macrophage, followed by CD45R/B220 (red) for B cells. Alternatively, the slides were sequentially incubated with CD3 (green) for T cells, followed by Gr-1 (red) for neutrophils (as detailed in materials and methods).. Slides were later stained with DAPI. Slides stained with isotype control mAb did not show any fluorescence (not depicted). Ten independent fields were photographed from each treatment at 100x magnification on a confocal microscope.

\section{Expression of mucosal integrin receptors on emTh cells in Bordetella infection}

Given that cell recruitment to the lung was delayed in B. pertussis infection (Fig. 1), we hypothesized that the timing of cell recruitment to the lung may be attributed to the phenotypic alteration of TRs on circulating and lung residential emTh cells. TRs were analyzed on emTh cells, defined as $\mathrm{CD} 44^{+} / \mathrm{CD} 45 \mathrm{RB}^{\text {low }}$ cells out of $\mathrm{CD} 4^{+}$cells as detailed in Fig. 2A.

In blood: Infection with $B$. parapertussis led to an immediate increase in circulating emTh cells that expressed $\alpha 4 \beta 7$ and $\alpha 4 \beta 1$ (39.3\% and 55.1\% respectively, Fig. 2B), which on average was 1.8-fold and 1.9-fold, respectively compared to similar cells from uninfected mice (Fig. 2C). However, circulating emTh cells derived from B. pertussis infection that expressed $\alpha 4 \beta 7$ and $\alpha 4 \beta 1$ was only $18.1 \%$ and $33.1 \%$, (Fig. 2B), which on average was 0.96-fold and 0.76-fold, respectively, compared to similar cells from uninfected mice (Fig. 2C).

At 25 days p.i. this pattern of expression was reversed and an increased proportion of circulating emTh cells derived from B. pertussis infection expressed $\alpha 4 \beta 7$ 
and $\alpha 4 \beta 1$ (35.2\% and 36.3\% respectively) (Fig. 2D) which was on average 1.8 -fold and 1.5-fold of similar cells from uninfected mice (Fig. 2E). Only $22.5 \%$ and $25.4 \%$ of respective cells were observed in $B$. parapertussis infection, which was similar to the frequency of these cells in uninfected mice (Figs 2D and 2E).

Blood emTh cells from Bp$\triangle P T X$ infection exhibited a similar pattern of $\alpha 4 \beta 7$ and $\alpha 4 \beta 1$ expression to that of B. parapertussis infection at both 5 days p.i. and 25 days p.i. (Figs A.3A and A.3B, see Appendix). No statistically significant differences were observed between blood emTh cells expressing CD11a and P-selectin ligand (P-sel lig) at either 5 or 25 days p.i. (Figs $2 \mathrm{C}$ and $2 \mathrm{E}$ ).

In lungs: In B. parapertussis infection, lung emTh cells displayed a TR phenotype similar to that found in uninfected samples at both 5 (Fig. 2G) and 25 days p.i. (Fig. 2I). In contrast, $41.7 \%$ and $42.2 \%$ of the lung emTh cells from B. pertussis infection expressed $\alpha 4 \beta 7$ and $\alpha 4 \beta 1$, respectively at 5 days p.i. (Fig. $2 \mathrm{~F}$ ), which on average were 1.37- and 1.14- fold, respectively, compared to similar cells from uninfected mice (Fig. 2G). At 25 days p.i. the frequency of lung emTh cells from B. pertussis infection that expressed $\alpha 4 \beta 7$ and $\alpha 4 \beta 1$ further increased to $42.1 \%$ and $55.4 \%$ (Fig. 2H), which were 2.19- and 2.17-fold, respectively, compared to similar cells from in uninfected mice (Fig. 2I). No statistically significant differences were observed between lung emTh cells expressing CD11a and P-sel lig at both 5 and 25 days p.i. (Figs $2 \mathrm{G}$ and 2I). Results obtained with $B p \triangle P T X$ closely resembled the results obtained with B. parapertussis (Figs A.3C and A.3D, see Appendix). 
2 A.

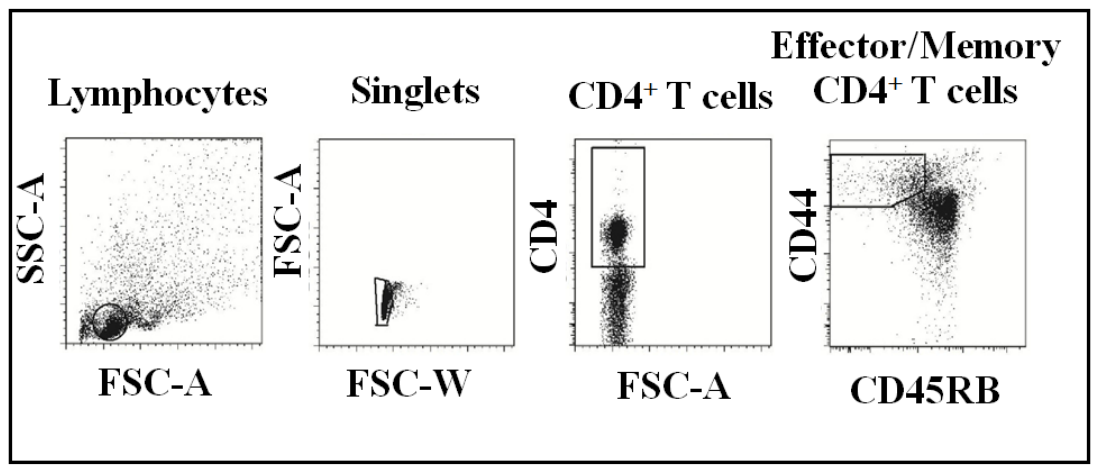

$2 B$.

Blood at 5 days p.i.

$2 D$.

Blood at 25 days p.i.
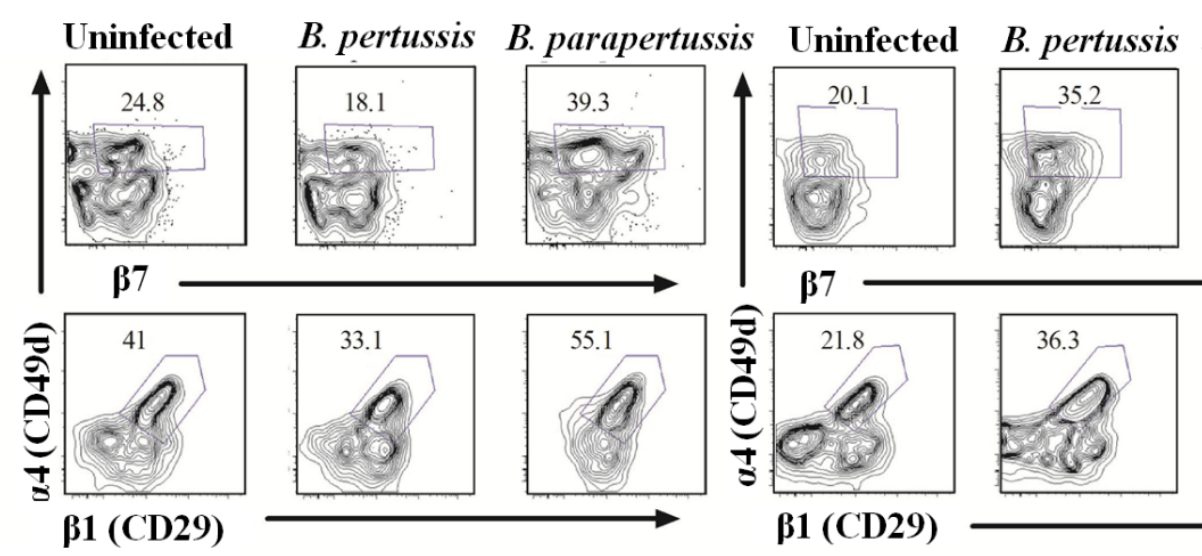

B. parapertussis

Blood at 5 days p.i.
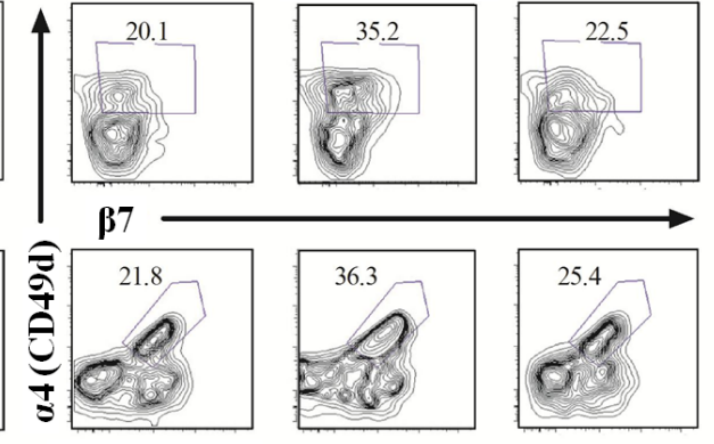

$2 \mathrm{C}$.

B1 (CD29)
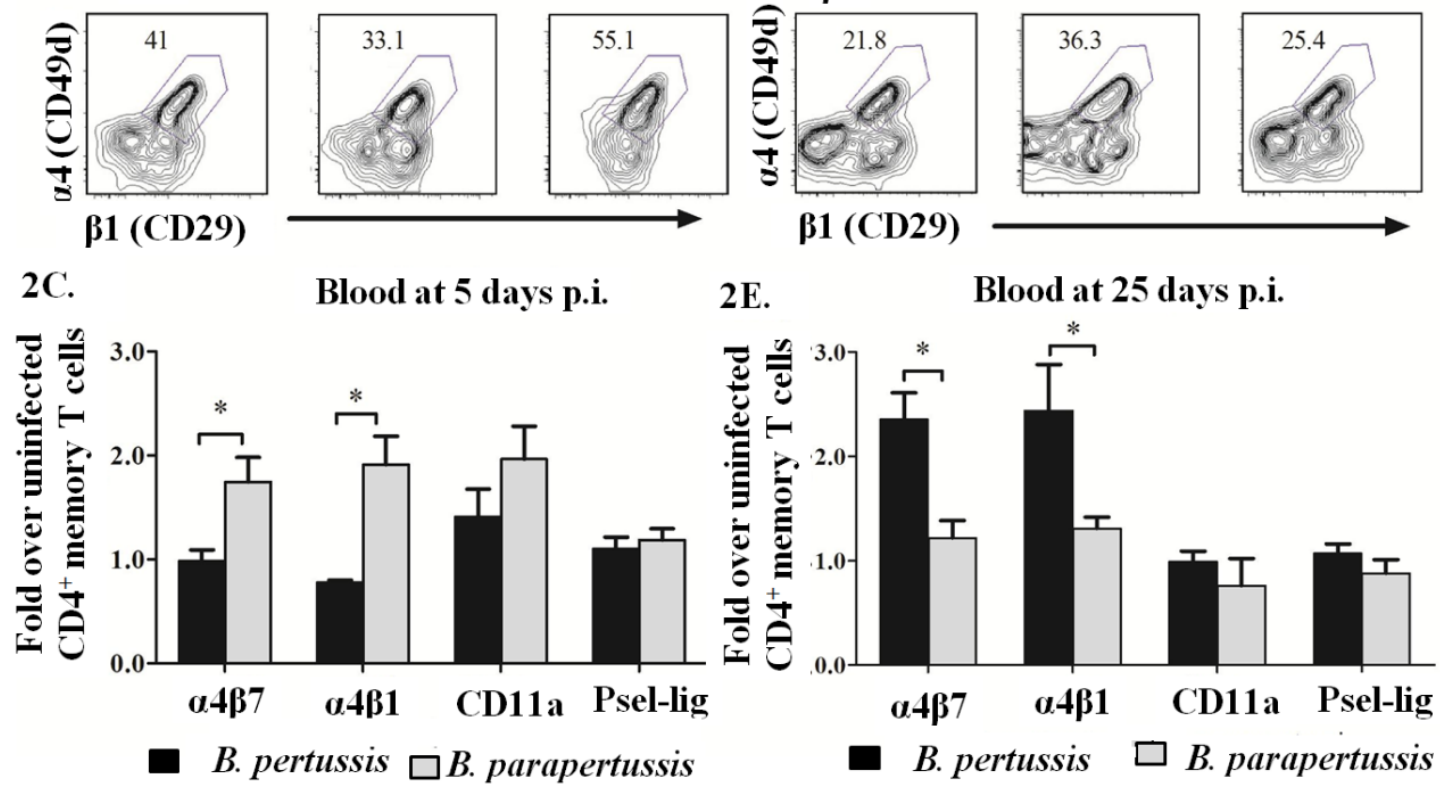
2H. Lung at 25 days p.i.

Uninfected B.pertussis B.parapertussis Uninfected

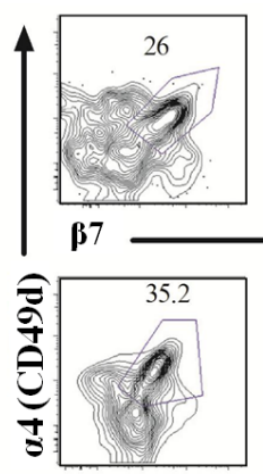

$\beta 1(C D 29)$
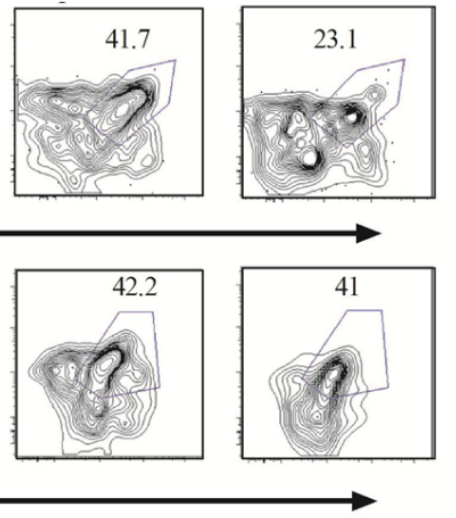

2G.

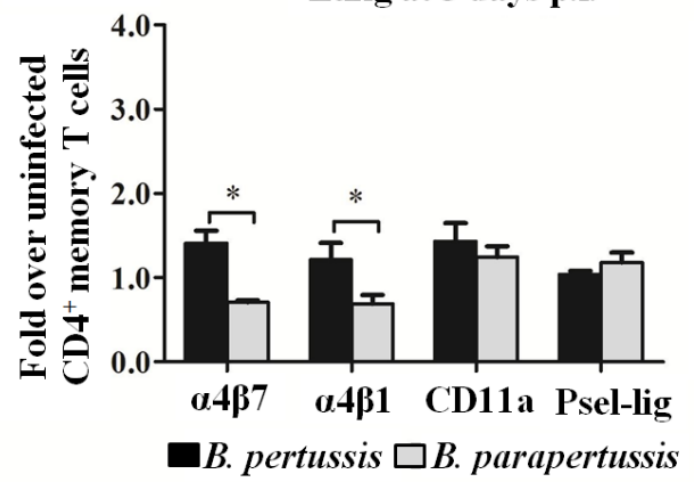

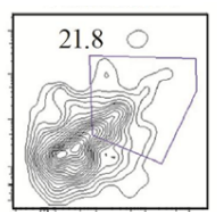

B. pertussis B. parapertussis
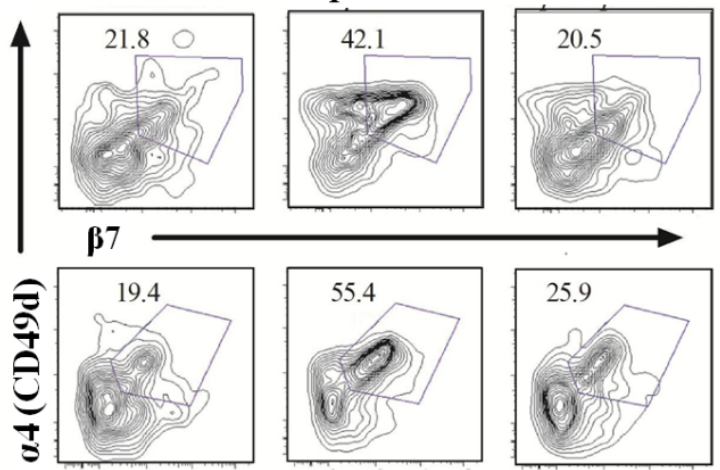

$\beta 1$ (CD29)

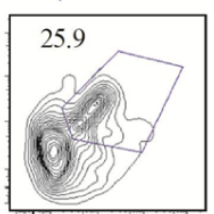

$2 I$.

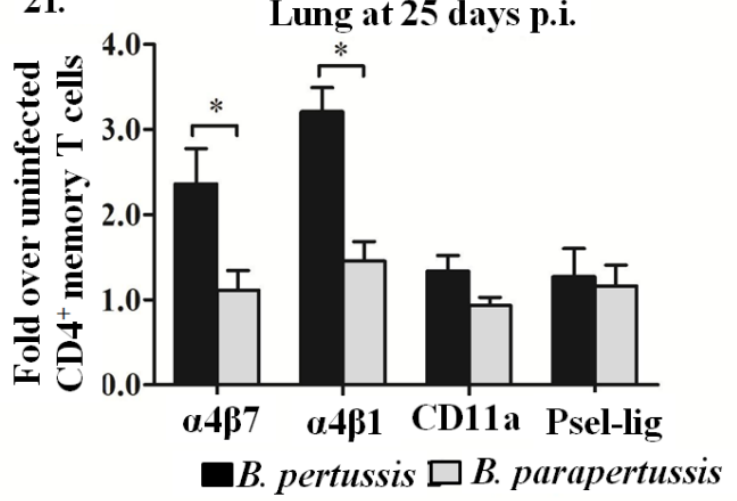

FIG. 2. Trafficking receptor expression on emTh cells in blood and lungs from B. pertussis- and B. parapertussis-infected mice at 5 and 25 days p.i. Mononuclear cells were obtained from the peripheral blood and lungs. Cells were gated for CD4 ${ }^{+}$ cells, and further gated for emTh cells $\left(\mathrm{CD} 44^{+} / \mathrm{CD} 45 \mathrm{RB}^{\text {low }}\right)$. (A) The frequency of emTh cells expressing TRs were assessed in blood $(\mathbf{B}, \mathbf{C}, \mathbf{D}, \mathbf{E})$ and lungs $(\mathbf{F}, \mathbf{G}, \mathbf{H}, \mathbf{I})$ and normalized to respective cells in uninfected mice (1.0 by definition). The percentages in the gating strategy are representatives from the group. Error bars represent SEM of four independent experiments $(n=4)$. *: $p<0.05$, no asterisk indicates $\mathrm{p} \square 0.05$ as analyzed by a two-tailed Student's $t$-test for comparison between B. pertussis- and B. parapertussisinfection. 


\section{In vitro migration and adhesion capabilities of blood leukocytes}

To further investigate the effect of PTX on the proportion of blood emTh cells that express $\alpha 4 \beta 7$ and $\alpha 4 \beta 1$ in $B$. pertussis infection, we assessed the ability of these cells to migrate or adhere under in vitro conditions. At 5 days p.i., compared to blood leukocytes from B. parapertussis-infected mice, $20 \%$ fewer blood leukocytes from B. pertussis-infected mice migrated to SDF-1a through the VCAM-1-coated transwells (Fig. 3A). Pretreatment of cells with PS/2 anti- $\alpha 4$ blocking antibody impeded the migration of leukocytes derived from B. pertussis- and B. parapertussis-infection, further demonstrating the role of $\alpha 4$ integrins in this process (Fig. 3A). Additionally, adherence of $B$. pertussis blood leukocytes to VCAM-1 coated surfaces was 53\% less than similar cells derived from B. parapertussis infection (Fig. 3B).

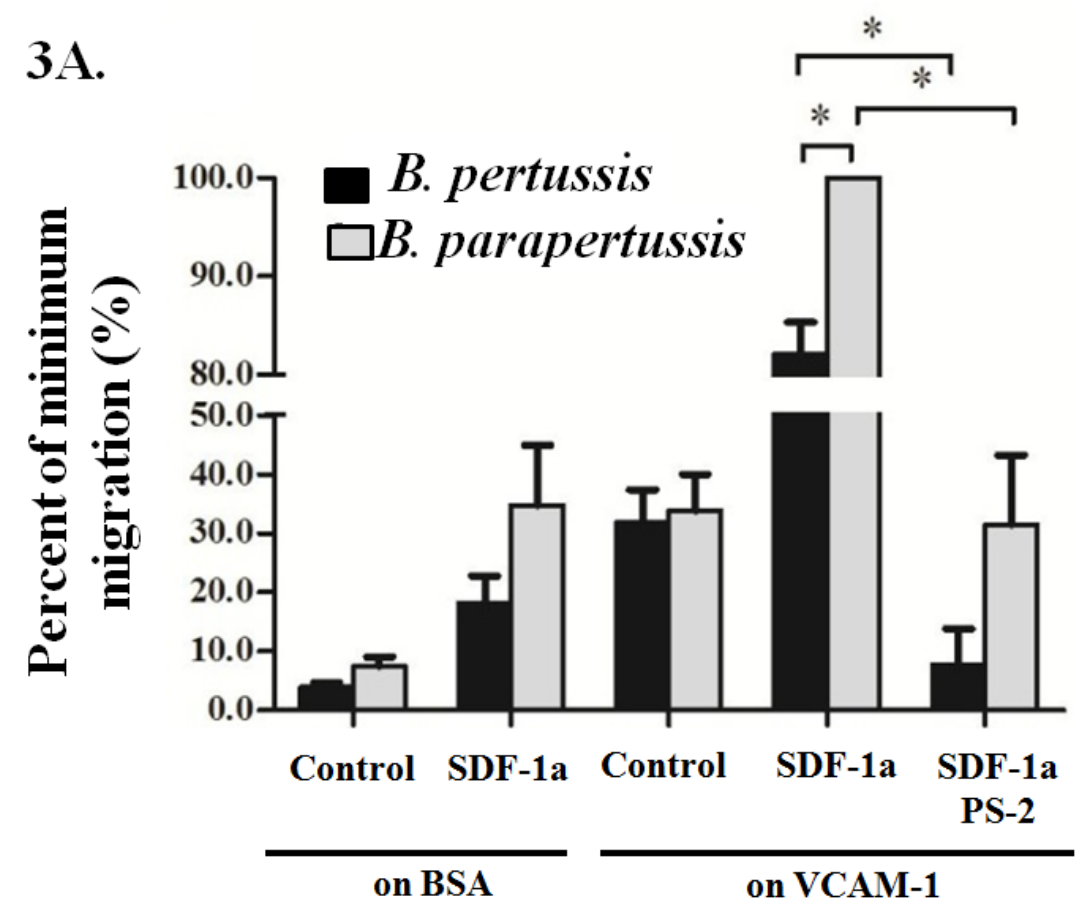


$3 B$.

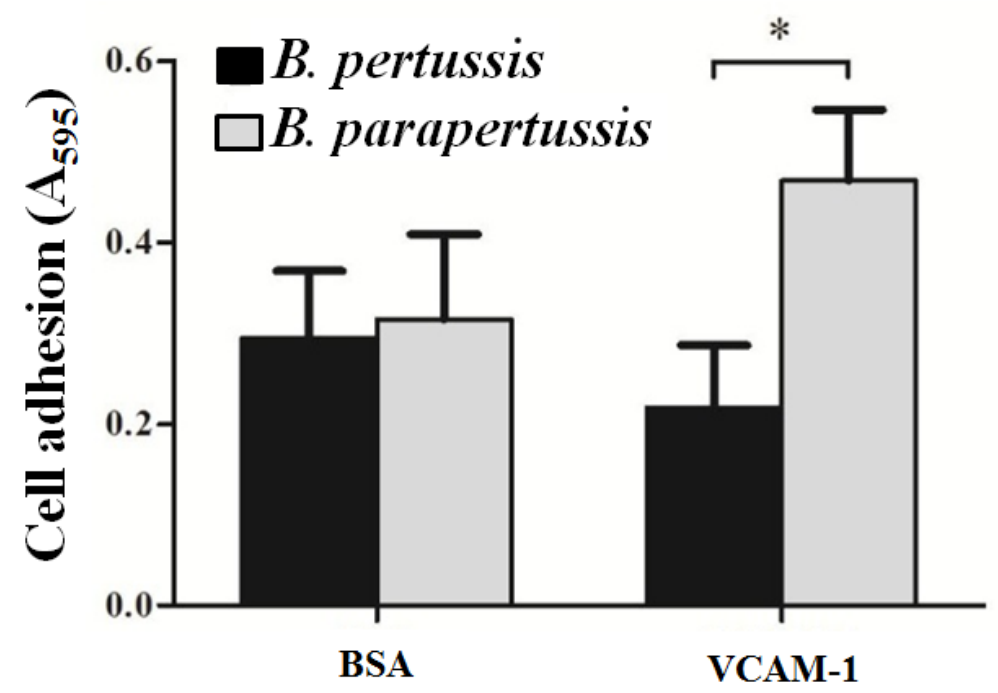

FIG. 3. Functional effects of $\alpha 4$ integrins on migration and adhesion of blood leukocytes from B. pertussis- and B. parapertussis-infection in vitro. (A) Migration of blood leukocytes from B. pertussis- and B. parapertussis-infection at 5 days p.i., through VCAM-1-coated or BSA-coated transwells in response to SDF-1a or control medium. Error bars represent the SEM of three independent experiments $(\mathrm{n}=3)$. A Student's $t$-test was performed. *: $\mathrm{p} \square 0.05$. (B) Adhesion of blood leukocytes at 5 days p.i. to 96-well plates pre-coated with VCAM-1 $(10 \mathrm{mg} / \mathrm{ml})$ or BSA, from B. pertussis- and B. parapertussis-infection. After 30 minutes, adherents were stained with crystal violet. Values represent mean absorbance at $\mathrm{A}_{595}$. Error bars represent the SEM of six independent experiments $(\mathrm{n}=6)$. A Student's $t$-test was performed. ${ }^{*}: \mathrm{p} \square 0.05$ between B. pertussis- and B. parapertussis-infection.

\section{Characterization of dendritic cell phenotype}

Since the imprinting of TRs on T cells is highly dependent on the maturation status and the regional and functional characteristics of the DCs (56), we next examined some of these parameters on lung DCs derived from mice infected with Bordetella strains. 
Lung mononuclear cells were gated on CD11 $\mathrm{c}^{+}$cells to select for DCs (Fig. 4A). A robust increase in the number of $\mathrm{CD} 11 \mathrm{c}^{+}$cells recruited to the lungs of mice infected with B. pertussis and B. parapertussis was observed at 5 days p.i. (Fig. A.4, see Appendix). A similar frequency of lung CD11 $\mathrm{c}^{+}$cells that expressed MHC class II, CD86, and CD11b were observed in B. pertussis- and B. parapertussis-infected mice, and those were 3.28-, 1.77-, and 7.21-fold in B. pertussis-infected mice and 3.28-, 1.75-, and 5.42-fold in B. parapertussis-infected mice, respectively, above $\mathrm{CD}_{11 \mathrm{c}^{+}}$cells derived from uninfected mice (Fig. 4B).

The proportion of CD11 $\mathrm{c}^{+}$cells that expressed CCR7 in B. pertussis-, and B. parapertussis-infected mice were 0.64-fold and 0.91-fold, respectively, compared to similar cells from uninfected mice (Fig. 4C). No significant difference was observed in the proportion of lung $\mathrm{CD} 11 \mathrm{c}^{+}$cells that expressed CXCR4 from the different infections (Fig. 4C). Lung CD11 $\mathrm{c}^{+}$cells in mice infected with Bp $\triangle P T X$ displayed similar maturation and GPCR patterns as B. parapertussis-infected mice (Fig. A.5, see Appendix).

At 25 days p.i. with B. parapertussis infection, the proportion of $\mathrm{CD} 11 \mathrm{c}^{+}$cells that expressed MHC class II, CD86, and CD11b was comparable to those in uninfected mice. In contrast, the proportion of $\mathrm{CD} 11 \mathrm{c}^{+}$cells expressing these markers from B. pertussis-infected mice that expressed these markers continued to be high and were 4.87-, 3.36-, and 3.48-fold, respectively, greater than similar cells from uninfected mice (Fig. 4D). No other statistically significant differences were observed between the infections for CCR7 and CXCR4 at 25 days p.i. (Fig. A.6, see Appendix). 
4 A.

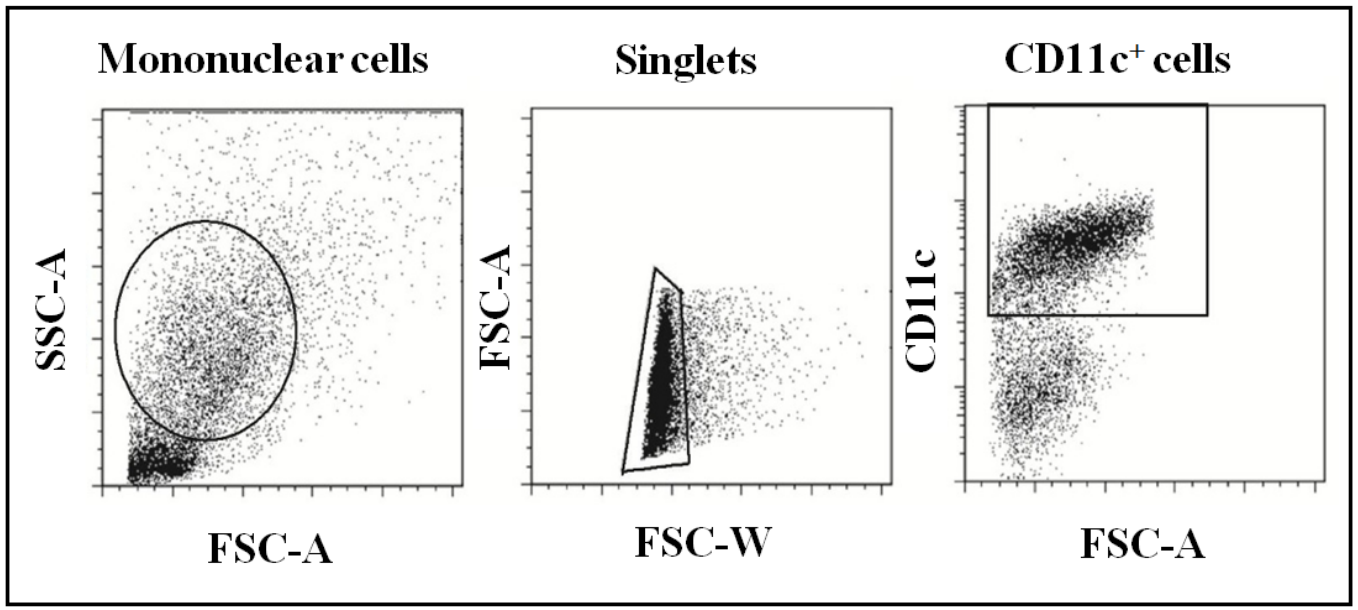

4B. Lung Dendritic cells at 5 days p.i.

4D. Lung Dendritic cells at 25 days p.i.
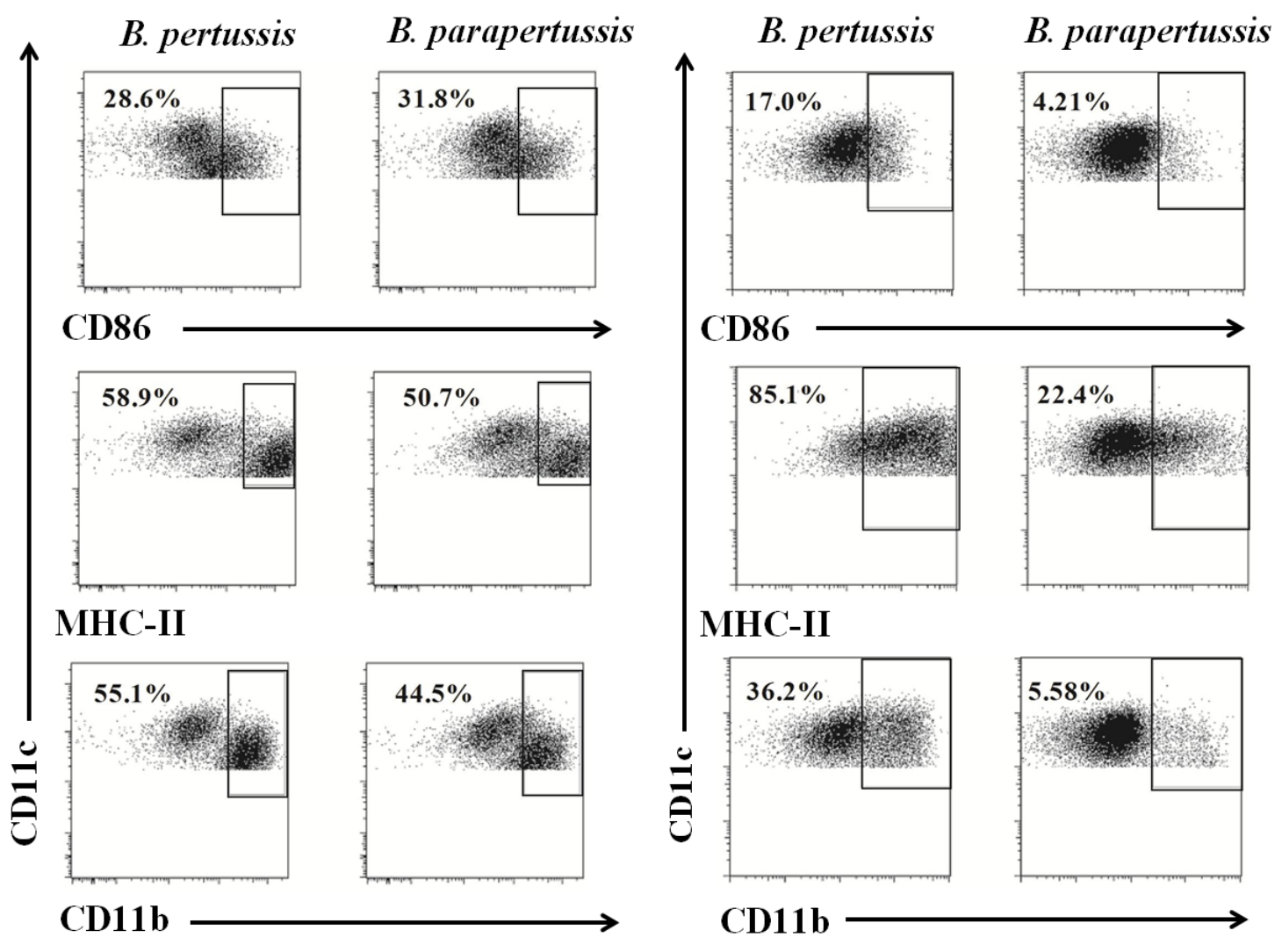
4C. Lung DC Maturation at 5 days 4E. Lung DC Maturation at 25 days

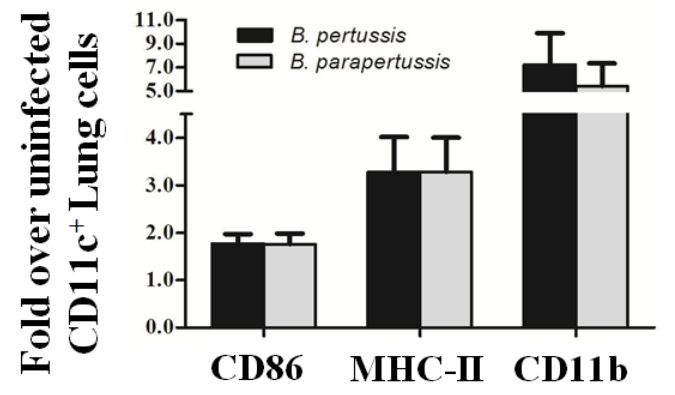

B. pertussis $\square$ B. parapertussis

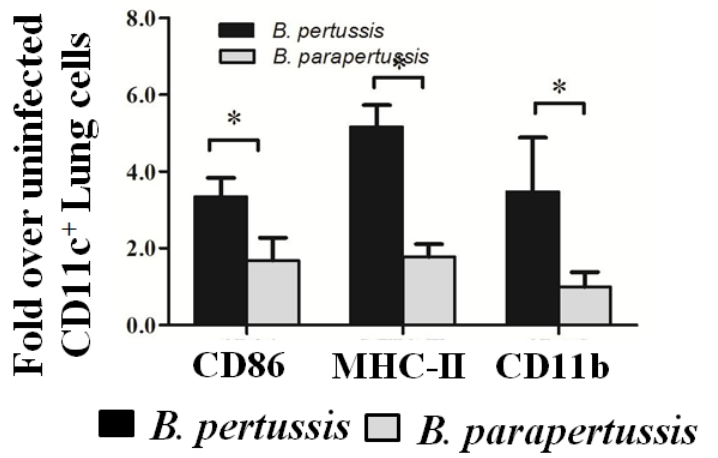

4F. Lung Dendritic cells at 5 days p.i.

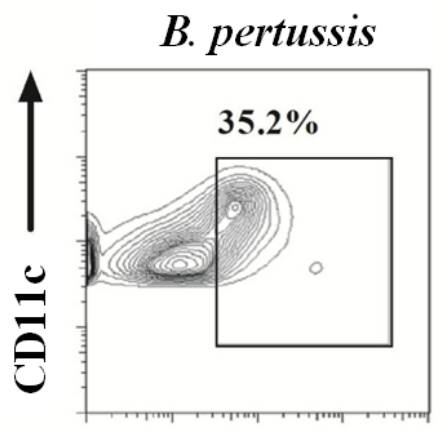

B. parapertussis

CCR7

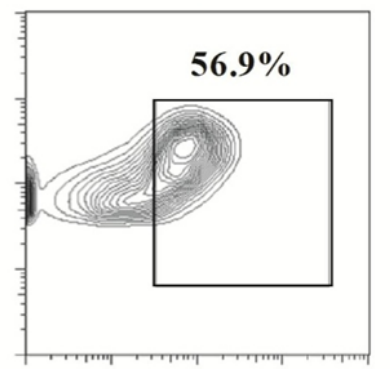

4G. Lung DC Trafficking Markers at 5 days p.i.

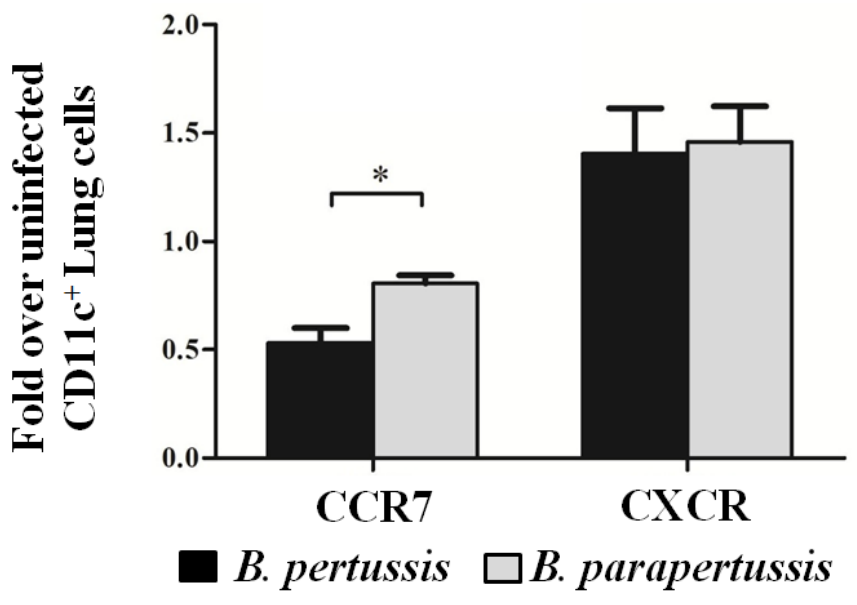


FIG. 4. Characterization of lung CD11 ${ }^{+}$cells during infection with $B$. pertussis and B. parapertussis. (A) Lung mononuclear cells were gated on singlets and then on CD11 ${ }^{+}$cells $(\mathrm{DCs})$. CD11 $\mathrm{c}^{+}$cells at 5 days p.i. (B, C) and 25 days p.i. (D, E) were analyzed for a second phenotypic marker as detailed in material and methods. Lung CD11c ${ }^{+}$cells expressing CCR 7 and CXCR 4 at 5 days p.i. $(\mathbf{F}, \mathbf{G})$ were measured. The percentages in the gating strategy are representatives from the group. Error bars represent the SEM of four independent experiments $(n=4)$. **: $p \llbracket 0.01, *: p \square 0.05$ and no asterisk: $\mathrm{p} \square 0.05$ as analyzed by a two-tailed Student's $t$-test.

\section{Trafficking receptor imprinting by Thy1.2 lung CD11 $\mathrm{c}^{+}$cells derived from Bordetella infection}

An allogenic system consisting of co-cultured Thy 1.2 lung $\mathrm{CD}_{11 \mathrm{c}^{+}}$cells derived from 5 days p.i. mice with naïve Thy $1.1 \mathrm{CD}^{+}$splenocytes was used to assess the TR imprinting. The proliferation of allogeneic Thy $1.1 \mathrm{CD}^{+}$cells was confirmed by a CFSE proliferation assay. Four generations of proliferation were observed in each treatment over a four-day period (Figs A.7A and 7B, see Appendix). Activated Thy $1.1 \mathrm{CD} 4^{+}$cells, designated by Thy $1.1^{+} / \mathrm{CD} 4^{+} / \mathrm{CD} 38^{++}$(Fig. 5A), were analyzed for the expression of TRs (Fig. 5B). Compared to uninfected cells, the proportion of activated Thy $1.1 \mathrm{CD} 4^{+}$cells that expressed $\alpha 4 \beta 7$ and $\alpha 4 \beta 1$ was 1.32-fold and 2.32-fold in B. parapertussis infection and 1.14- and 1.03-fold in Bp $\triangle P T X$ infection, respectively (Fig. 5B). However, in the coculture systems comprising of $\mathrm{CD} 11 \mathrm{c}^{+}$cells from $B$. pertussis infection the proportion of $\alpha 4 \beta 7$ and $\alpha 4 \beta 1$ expressing Thy $1.1^{+} / \mathrm{CD} 4^{+} / \mathrm{CD} 38^{++}$cells was 0.77 -fold and 0.46 -fold, respectively, compared to similar cells from uninfected system (Fig. 5B). 
$5 \mathrm{~A}$.

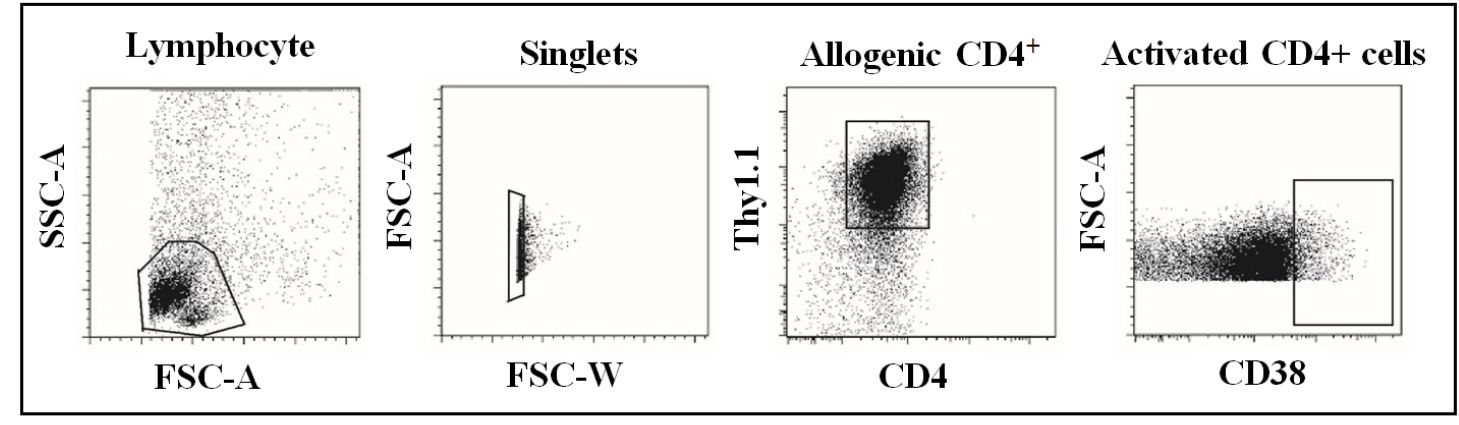

5B. DC Imprinting at 5 days p.i

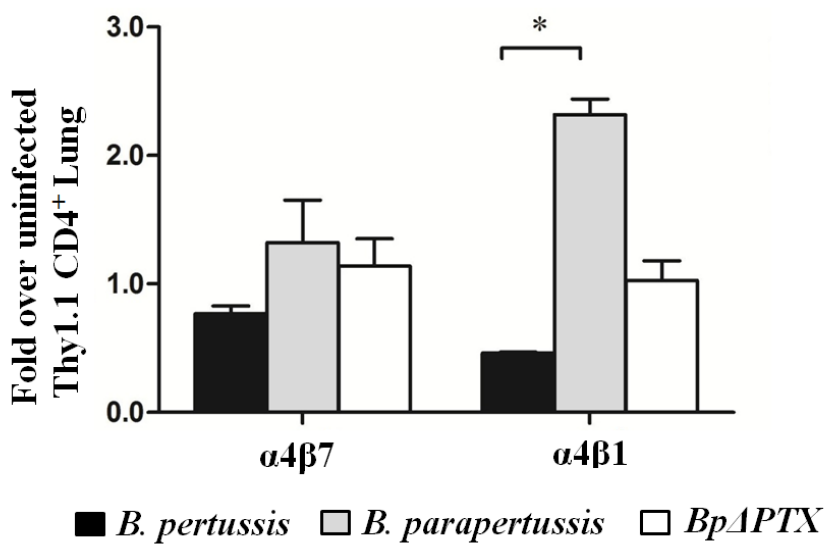

FIG. 5. $\alpha 4 \beta 7$ and $\alpha 4 \beta 1$ imprinting on allogenic $\mathrm{Thy} 1.1^{+} / \mathrm{CD}^{+} / \mathrm{CD}^{+++}$cells. Lung DCs at 5 days p.i. from $B$. pertussis, B. parapertussis, Bp $\triangle P T X$, or uninfected, were cocultured for four days with naïve allogeneic Thy1.1 CD4 ${ }^{+}$splenocytes at 1:5 ratio (A) Co-cultured cells were gated on lymphocytes, followed by singlets and then on Thy $1.1^{+} / \mathrm{CD} 4^{+} / \mathrm{CD} 38^{++}$cells. (B) Proportion of Thy $1.1^{+} / \mathrm{CD} 4^{+} / \mathrm{CD} 38^{++}$cells expressing $\alpha 4 \beta 7$ or $\alpha 4 \beta 1$ following co-culture with lung DCs-derived from Bordetella infections (as detailed in material and methods). Error bars represent the SEM of three independent experiments $(\mathrm{n}=3)$. *: $\mathrm{p} \leq 0.05$, no asterisk: $\mathrm{p} \square 0.05$ for the comparison of $B$. pertussis and $B$. parapertussis derived cells as analyzed by a two-tailed Student's $t$-test. 


\section{DISCUSSION}

Microbial pathogens, such as Bordetella, utilize virulence factors to interfere with various immune functions to promote their pathogenesis and prolong their survival in the host (13), (41), (24), (11), (2). The combined expression of integrin receptors and chemokine receptors on leukocytes direct them to specific organs and lymph nodes (14). However, integrin receptors and adhesion molecules are utilized as targets for entry and cellular signaling during bacterial and viral infections, for instance in Staphyloccocus aureus and Neisseria species (27) and by human cytomegalovirus and rotaviruses, respectively (59). Yet, the interference of pathogens with the imprinting of integrin receptors on lymphocytes as a strategy to divert or delay immune cell migration to infected organs is not well documented. This study tested the hypothesis that during infection with $B$. pertussis, despite the extreme lymphocytosis observed immediately after infection, the circulating lymphocytes display a compromised integrin receptor expression pattern due to imprinting defects induced by pertussis toxin (PTX).

Although B. pertussis and Bordetella parapertussis (B. parapertussis) are natural human pathogens, experimental infections in mice closely mimic human pathology, and considerable information on Bordetella pathogenesis and studies on Bordetella vaccine is based on mouse models (49). Therefore using a BALB/c mouse model, we compared infection by $B$. pertussis with infection by $B$. parapertussis - a family member that does not express PTX, but otherwise demonstrates 90\% genome homology with B. pertussis (29). Unlike $B$ pertussis, $B$. parapertussis induces a mild respiratory infection that is quickly resolved by the immune system (7), indicating that PTX is essential for bacterial 
colonization. We tested our main hypothesis regarding PTX using these two natural human pathogens and then confirmed our results using Bp$\triangle P T X$, a pertussis strain that cannot produce PTX. Two time points post-infection were assessed throughout the study to cover both the effector and memory immune responses. At 5 days p.i., maximum numbers of bacteria were isolated from the lungs (Fig. A1), and blood leukocytes peaked in all infectious models (Fig. A2). At 25 days p.i., no bacteria were isolated from the lungs and blood leukocytes dropped in all infectious models. These time points, respectively, defined the early and late phases of the infection.

$\alpha 4 \beta 7$ and $\alpha 4 \beta 1$ integrins play an important role in lymphocytes migration to mucosal tissues such as gut and lungs (42), (47). Therefore, the analyses in this study were focussed on $\alpha 4 \beta 7$ - and $\alpha 4 \beta 1$-expressing emTh cells, assuming that the majority of these activated cells are Bordetella specific. Further study would be required to substantiate this assumption.

Mainly neutrophils and few lymphocytes were recruited at 5 days p.i. in B. parapertussis- and $B p \triangle P T X$ - infected lungs. Cell recruitment and subsequent inflammation coincided with a rise in the frequency of circulating $\alpha 4 \beta 7$ - and $\alpha 4 \beta 1$ expressing emTh cells. In contrast, there was no increase in leukocyte recruitment to the lungs of mice infected with $B$. pertussis at 5 days p.i., a result previously reported by others (3). Despite peripheral blood lymphocytosis in the B. pertussis infection model, a reduced frequency of circulating $\alpha 4 \beta 7$ - and $\alpha 4 \beta 1$-expressing emTh cells was detected, suggesting that these lymphocytes have a limited capacity to reach the infected lungs. However, a modest increase in the frequency of $\alpha 4 \beta 7$ - and $\alpha 4 \beta 1$-expressing $\mathrm{CD} 4^{+}$cells in 
the lungs was observed, which may be an indicative of local T cell activation and imprinting by resident mature lung DCs, due to persistent exposure to B. pertussis antigens (31), (33).

No signs of lung inflammation were observed in B. parapertussis or Bp$\triangle P T X$ infected mice at 25 days p.i., and the frequency of circulating emTh cells expressing $\alpha 4 \beta 7$ and $\alpha 4 \beta 1$ dropped to levels similar to those in uninfected mice, suggesting complete resolution of infection. Surprisingly, only at 25 days p.i was extensive inflammation and massive lymphocyte recruitment observed in lungs of mice infected with B. pertussis, corresponding with an increase in both circulating and lung resident $\alpha 4 \beta 7$ - and $\alpha 4 \beta 1$ expressing emTh cells. This implies a delay of several weeks in the adaptive immune responses to $B$. pertussis respiratory infection.

Fedele et al. (23), (22) associated the late Th17 responses in B. pertussis infection, but not in B. parapertussis infection, to the effects of LPS and adenylate cyclase on DC, and Carbonetti et al. (5) demonstrated that late Th17 responses contributed to the eventual resolution of $B$. pertussis infection in experimental mice. Still to be determined is whether $\alpha 4 \beta 7$ - and $\alpha 4 \beta 1$-expressing emTh cells secrete IL-17. If so, this may additionally confirm a late role for Th17 cells in the resolution of $B$. pertussis infection.

VCAM-1, an adhesion molecule expressed on vascular endothelial cells in the lungs (51), is a common ligand for both $\alpha 4 \beta 1$ and $\alpha 4 \beta 7$ (50). SDF-1 is a highly effective lymphocyte chemoattractant (10). Our in vitro assays using B. parapertussis and B. pertussis infection models revealed a $\alpha 4$-dependent migration, to SDF-1 through VCAM-1. . However, lymphocytes derived from B. pertussis infection model displayed 
a reduced binding to VCAM-1 surfaces and also exhibited a decreased $\alpha 4$-dependent migration towards SDF-1, compared to lymphocytes derived from B. parapertussisinfected mice. These results indicate an effect of PTX on $\alpha 4$-integrins.

TR imprinting is a function of the DC maturation status, origin and phenotype (56). In contrast to the report of partial DC maturation during B. pertussis infection (52), our results indicate that at 5 days p.i. a comparable percentage of lung DCs derived from all three infection models upregulated MHC class II and CD86 suggesting that DC maturation is PTX independent. Furthermore, the expression of these markers persisted in $B$. pertussis infection at 25 days p.i., but not in the other models of infection, suggesting a prolonged exposure of lung DCs to B. pertussis-antigens.

Integrin $\alpha \mathrm{M} \beta 2$ (CD11b/CD18)is mainly expressed on leukocytes and $\alpha \mathrm{M}$ plays a pivotal role in adhesion and spreading of these cells on $\alpha \mathrm{M} \beta 2$ ligands (fibrinogen, Factor $\mathrm{X}, \mathrm{iC} 3 \mathrm{~b}, \mathrm{ICAM}-1)$ (57). It has been reported that adenylate cyclase toxin (ACT) binds to $\alpha \mathrm{M} \beta 2$-expressing immune cells, including DCs via this integrin receptor (26), and promotes internalization of ACT and integrins(38). However, our results showed that at 5 days p.i. a comparable proportion of lung DC derived from all three Bordetella infections upregulated $\alpha \mathrm{M}$, but this expression persisted at 25 days p.i. only in B. pertussis infection, suggesting a prolonged exposure of lung DCs to B. pertussis antigens. The difference in results observed between our studies may be due to the use of whole Bordetella bacterium for infection in our experiments verses the use of recombinant ACT by others. 
The expression of CCR7 on mature DCs is required for their exit from the peripheral tissues and entrance into the lymphatics (20), (36), (53). Despite an increased percentage of mature DCs in the lungs of B. pertussis-infected mice, the frequency of $\mathrm{CCR}^{+}$DCs was reduced compared to B. parapertussis-infected mice. This suggests that in B. pertussis infection lung DCs may be subjected to PTX-A intoxication and GPCR inhibition, leading to reduced accessibility to secondary lymphoid organs. Although CXCR4 has been suggested to contribute to DC trafficking between tissues and lymphatics (32), no significant differences in the percentage of DCs expressing CXCR4 were observed between our infection models, implying that this GPCR plays a limited role in these models.

The imprinting capabilities of lung-derived DCs from Bordetella infections were assessed in an allogeneic system. Lung DCs from all Bordetella infection models expressed maturation markers at 5 days p.i., and induced equal proliferation of allogeneic Thy1.1 CD4 ${ }^{+}$cells in a four-day co-culture (Fig. A.7B). However, the percentage of allogeneic $\mathrm{CD}^{+}$cells that expressed $\alpha 4 \beta 1$ was significantly reduced in the co-culture system of lung DCs from B. pertussis-infected mice compared to the same system stimulated with lung DCs from B. parapertussis- or Bp $\triangle P T X$-infected mice. In this experiment, $\alpha 4 \beta 7$ imprinting was not different between allogeneic systems, suggesting that additional mechanisms may contribute to PTX-dependent $\alpha 4 \beta 7$ expression on emTh cells.

Of note, the respiratory disease was induced intranasally in mice; this route of infection may have caused a possible leakage of Bordetella bacteria/antigens into the 
esophagus in addition to delivery through the trachea. In this circumstance Bordetella antigens may be presented in both gut associated lymphoid tissues by gut-derived DCs that imprint $\alpha 4 \beta 7$-expressing emTh cells, as well as in bronchial associated lymphoid tissues by lung-derived DCs that primarily imprint $\alpha 4 \beta 1$-expressing emTh cells. The experimental setting presented in Fig.5 reflects only lung-associated DC imprinting, and thus imprinting primarily of $\alpha 4 \beta 1$-expressing emTh cells.

The results of this study do not rule out the possibility that soluble PTX may diffuse into respiratory draining lymph nodes, triggering a pre-imprinted lymphocyte egress in an S1P-1 (Sphingosine 1 Phosphate receptor 1)-independent manner, as previously suggested (46). Assessment of this prospect would require further study. Although we focus on PTX-dependent effects on $\alpha 4 \beta 7$ - and $\alpha 4 \beta 1$-imprinting on emTh cells, other TRs may play an important role in immune cell homing to the lung during respiratory infection, and it is our intent to further explore this prospect.

In conclusion, the results of this study suggest that $\alpha 4$ integrins, in combination with $\beta 7$ and $\beta 1$, play an important role in emTh cell migration to infected lungs. B. pertussis alters the expression of these TRs in a PTX-dependent manner, and thus delays a $\mathrm{T}$ cell mediated immune response that would otherwise promptly resolve the infection. Mechanisms of selective trafficking of lymphocytes to respiratory tissues are likely to be critical in the immune response to airborne agents. Thus, the imprinting and expression of mucosal- and lung-associated trafficking molecules should be addressed in the design of vaccines directed against respiratory pathogens. 


\section{AKNOWLEDGEMENTS}

This work was supported by funds from CSU research award and National

Institutes of Health grant (1SC3GM100854-01). 
CHAPTER III

CONCLUSION 


\section{CONCLUSION}

It is a paradox that during $B$. pertussis infection, although pertussis toxin induces extreme lymphocytosis, recovery is delayed up to several weeks. DC characteristic and phenotype are vital to the nature of trafficking receptors imprinted on $\mathrm{T}$ cells. Therefore, in this study we investigated the effect that PTX has on DC ability to imprint lungassociated trafficking receptors on $\mathrm{T}$ cells. While there are a number of trafficking receptors documented to be important for lung trafficking, this study focused on $\alpha 4 \beta 7$ and $\alpha 4 \beta 1$ because these integrins show appreciable changes in response to Bordetella infections. The results of this study indicate that although PTX does not modify DC maturation, lung-associated trafficking receptor imprinting on $\mathrm{T}$ cells is diminished in B. pertussis infection. This may explain the prolonged disease and delayed resolution of B. pertussis infections.

\section{Future Directions}

- Late Th17 responses in B. pertussis infection were previously demonstrated and suggested to be responsible for the eventual resolution of the infection in mice. Still to be determined is whether $\alpha 4 \beta 7$ and $\alpha 4 \beta 1$ expressing emTh cells secrete IL-17; if so, this may further confirm a late role for Th17 cells in the resolution of B. pertussis infection (5).

- Still to be explored is whether soluble PTX diffuses into the lymphatics and respiratory draining lymph nodes, triggering a pre-imprinted lymphocyte egress in an S1P (Sphingosine 1 Phosphate receptor 1)-independent manner, as previously suggested (46). 
- In the future we plan to substantiate the contribution of $\alpha 4 \beta 7$ and $\alpha 4 \beta 1$ receptors to emTh cell-homing during Bordetella-infection by injecting mice with anti- $\alpha 4$ PS/2 antibody prior to adaptive transfer of CFSE-stained peripheral blood mononuclear cells (34).

- Although we focused on PTX-dependent effects on $\alpha 4 \beta 7$ and $\alpha 4 \beta 1$ imprinting on effector/memory $\mathrm{CD}^{+}$cells, other TRs may play an important role in immune cell homing to the lung during respiratory infection, and it is our intent to further explore this field in the future studies. The discovery of additional TR that are more exclusive to lung trafficking will aid in the development of vaccines against respiratory pathogens. 


\section{APPENDIX: SUPPLEMENTARY DATA}

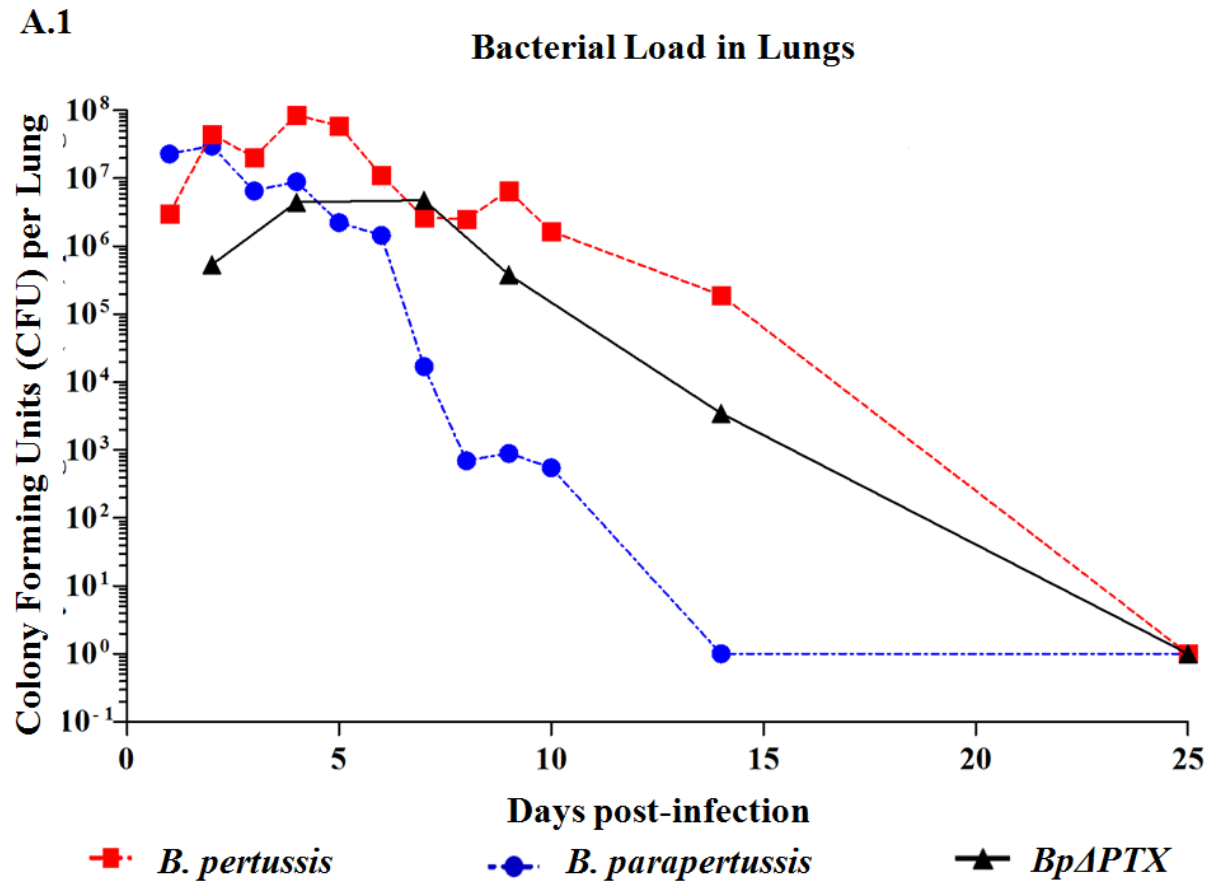

FIG. A.1. B. pertussis induces a longer course of infection than $B$. parapertussis or Bp $\triangle P T X$. BALB/c mice were infected i.n. with $5 \times 10^{6} \mathrm{CFU}$ of Bordetella strains in $20 \mu \mathrm{l}$ PBS or mock infection (as detailed in material and methods). Bacterial load in the lungs was assessed at the time points shown above. This graph represents data from one of two independent experiments. Additionally, bacterial load in the lungs at day 3 p.i. and day 23 p.i. were assessed in each of the experiments conducted (data not shown). 
A.2

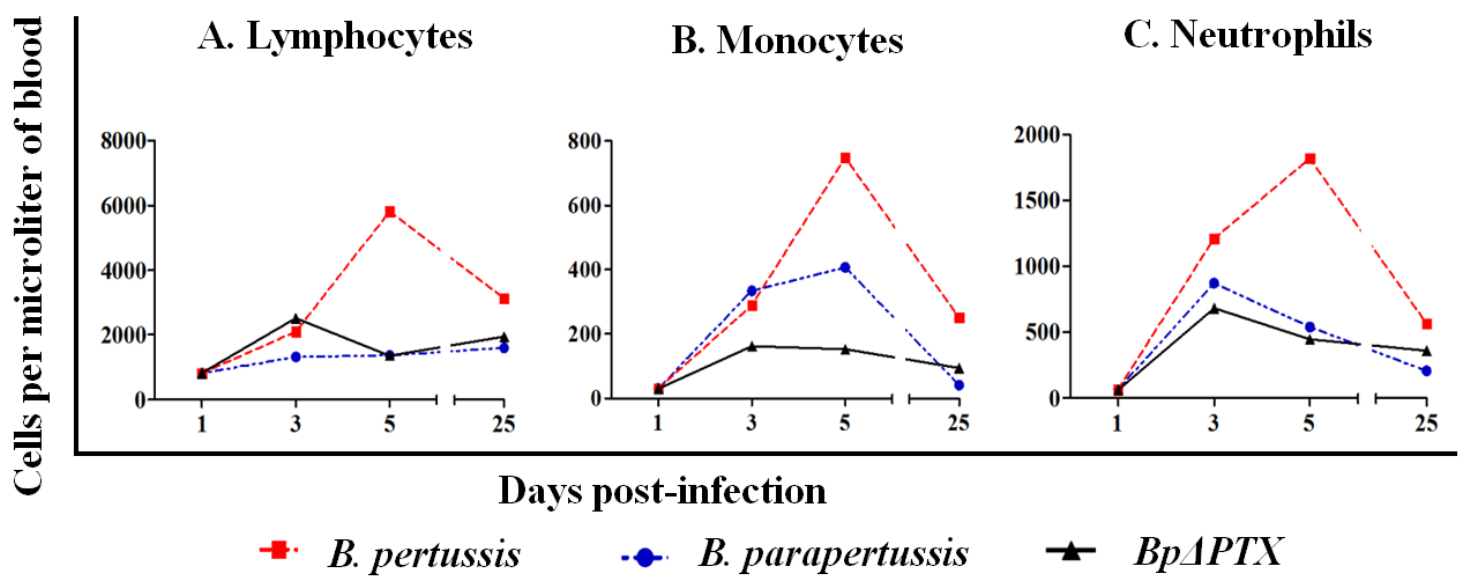

FIG. A.2. Blood immunophenotyping during the course of Bordetella infections. Absolute numbers of circulating (A) lymphocytes, (B) monocytes and (C) neutrophils were estimated by flow cytometry using trucount beads (as detailed in material and methods). These graphs represent one of two independent experiments. 
A.3A Blood at 5 days p.i.

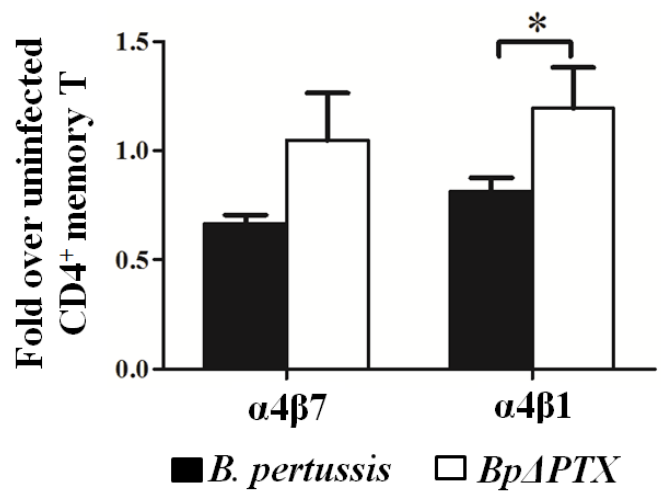

A.3C Lung at 5 days p.i.

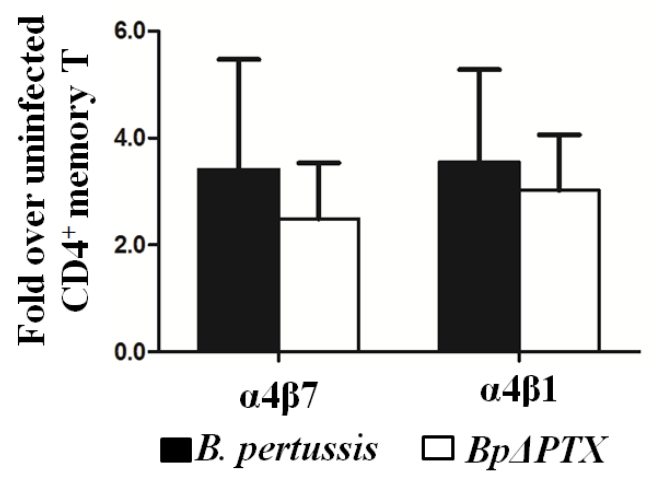

A.3B Blood at 25 days p.i.
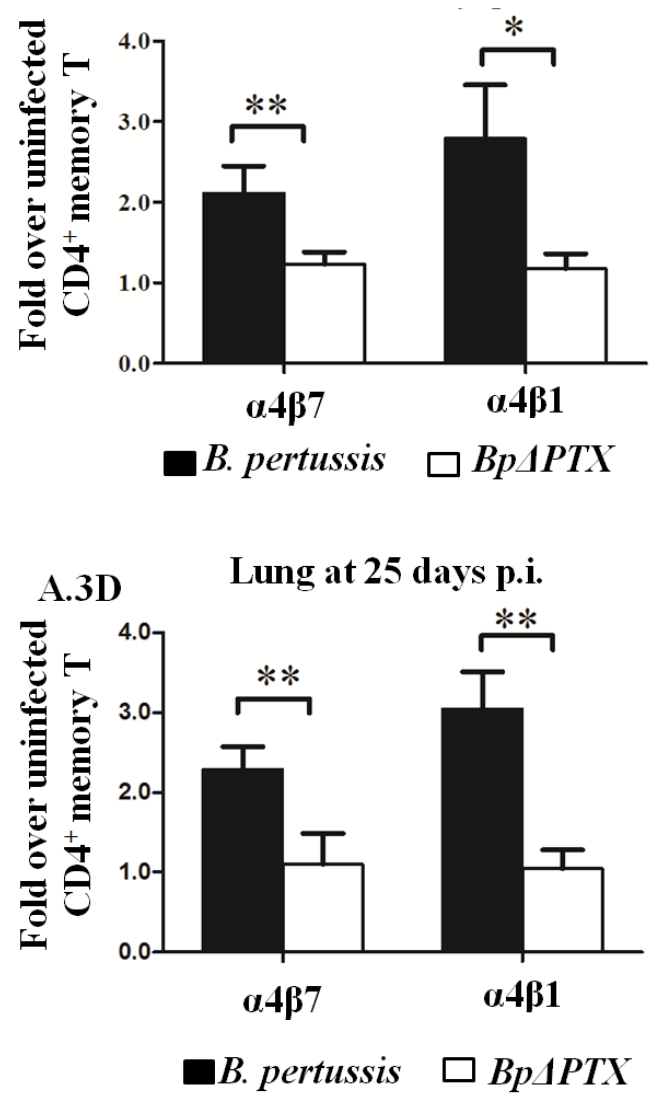

FIG. A.3. Comparative trafficking receptor (TR) expression on emTh cells between B. pertussis and BpAPTX infections. Mononuclear cells were obtained at 5 and 25 days p.i. from peripheral blood and from perfused lungs. Isolated cells were stained with conjugated antibodies (as detailed in material and methods) and acquired by flow cytometer. Mononuclear cells were gated for $\mathrm{CD} 4^{+}$cells, and further gated for emTh cells $\left(\mathrm{CD} 44^{+} / \mathrm{CD} 45 \mathrm{RB}^{\text {low }}\right)$ as shown in Fig. 2A. The percent of emTh expressing TRs from blood (A, B) and lungs (C, D) were assessed. The cell populations of interest were normalized to populations from uninfected mice (1.0 by definition). Error bars represent SEM from three to four independent experiments. *: $\mathrm{p}<0.1, * *$ : $\mathrm{p}<0.05$, no asterisk indicates $\mathrm{p} \square 0.1$ as analyzed by a two tailed Student's $t$-test for comparison between $B$. pertussis and $B p \triangle P T X$ treatments. 


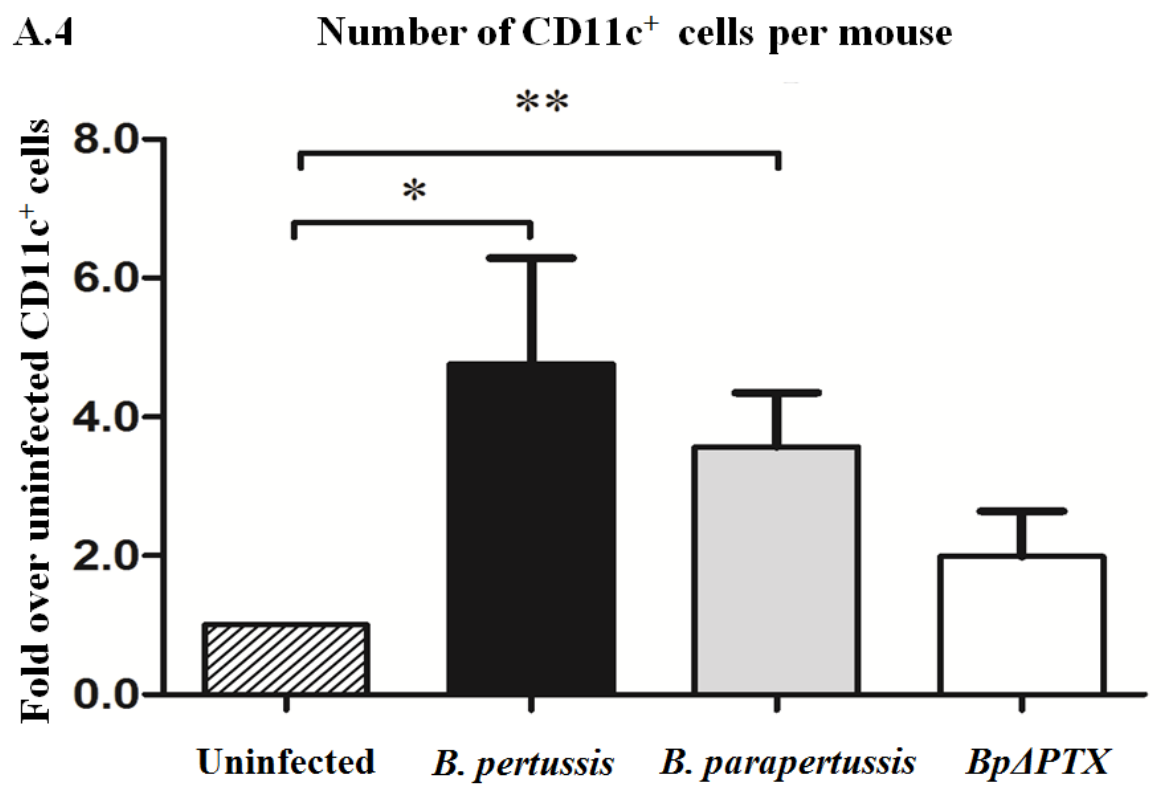

FIG. A.4. Drastic increase of $\mathrm{CD}_{11} \mathrm{c}^{+}$cells during the early phase of infection with Bordetella strains. Mononuclear cells were isolated from lungs and enriched for DC $\left(\mathrm{CD} 11 \mathrm{c}^{+}\right)$as detailed in material and methods. After the column enrichment, the total number of $\mathrm{CD} 11 \mathrm{c}^{+}$cells per mouse was estimated using the hemocytometer. The cell populations of interest were normalized to populations from uninfected mice (1.0 by definition). Error bars represent SEM from three to five independent experiments. *: $\mathrm{p}$ $<0.1, * *$ : $\mathrm{p}<0.05$, no asterisk indicates $\mathrm{p} \square 0.1$ as analyzed by a two tailed Student's $t$-test. 

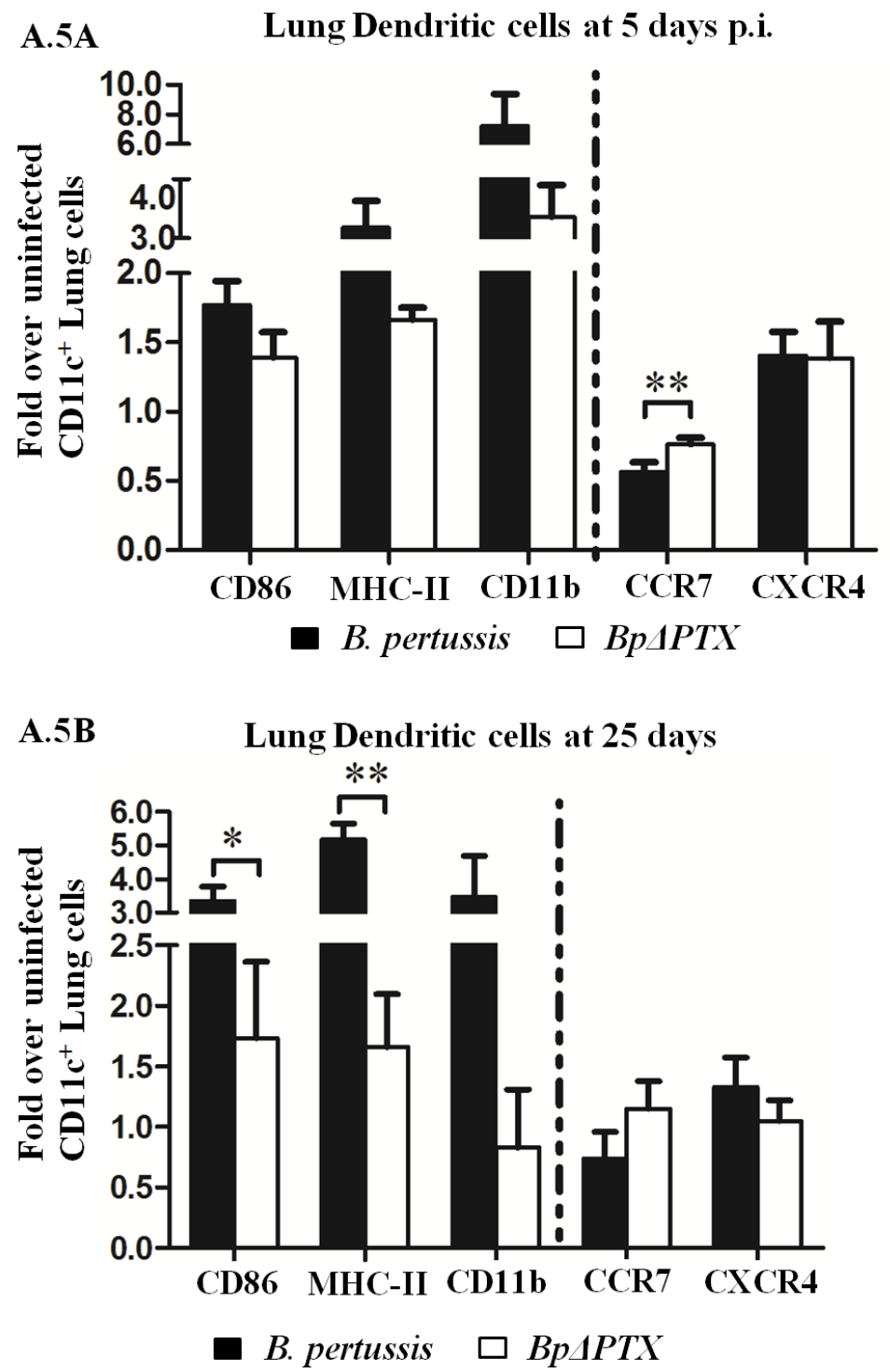

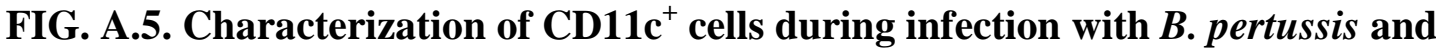
BpAPTX. Mononuclear cells were obtained at 5 and 25 days p.i. from perfused lungs. Isolated cells were stained with conjugated antibodies (as detailed in material and methods) and acquired on a flow cytometer. Mononuclear cells were gated for $\mathrm{CD} 11 \mathrm{c}^{+}$ cells as shown in Fig. 4A. CD11 $\mathrm{c}^{+}$cells (DCs) were analyzed for a second phenotypic marker. The number of matured dendritic cells expressing CD86, MHC-class II, CD11b, CCR7, and CXCR4 at (A) 5 days p.i and (B) 25 days p.i. were measured. Data represent the mean of three independent experiments $(n=3)$. Error bars represent SEM. $*$ : $p<0.1$, **: $\mathrm{p}<0.05$, no asterisk indicates $\mathrm{p} \square 0.1$ as analyzed by a two tailed Student's $t$-test for comparison between $B$. pertussis and $B p \triangle P T X$ treatments. 


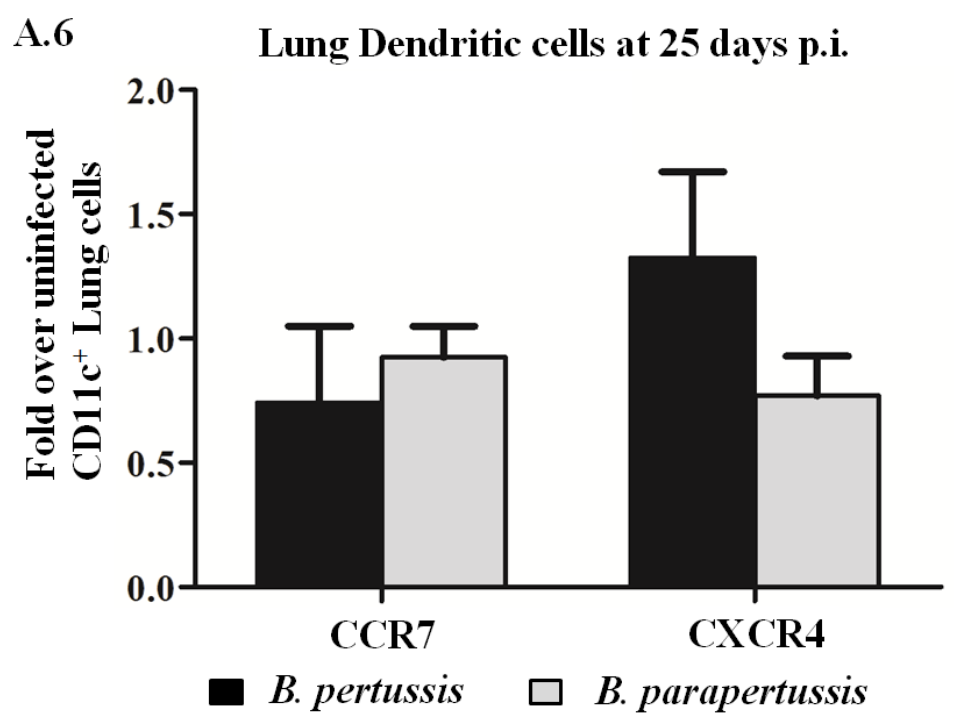

FIG. A.6. Characterization of maturation markers on $\mathrm{CD11c}^{+}$cells in B. pertussisand $B$. parapertussis-infected mice at 25 days p.i. Mononuclear cells were obtained at 25 days p.i. from perfused lungs. Isolated cells were stained with conjugated antibodies (as detailed in material and methods) and acquired on a flow cytometer. Mononuclear cells were gated for $\mathrm{CD} 11 \mathrm{c}^{+}$cells as shown in Fig. 4A. Error bars represent the SEM of two independent experiments $(n=2)$. Data were analyzed using two-tails Student's t-test. No asterisk indicates $\mathrm{p} \square 0.05$. 


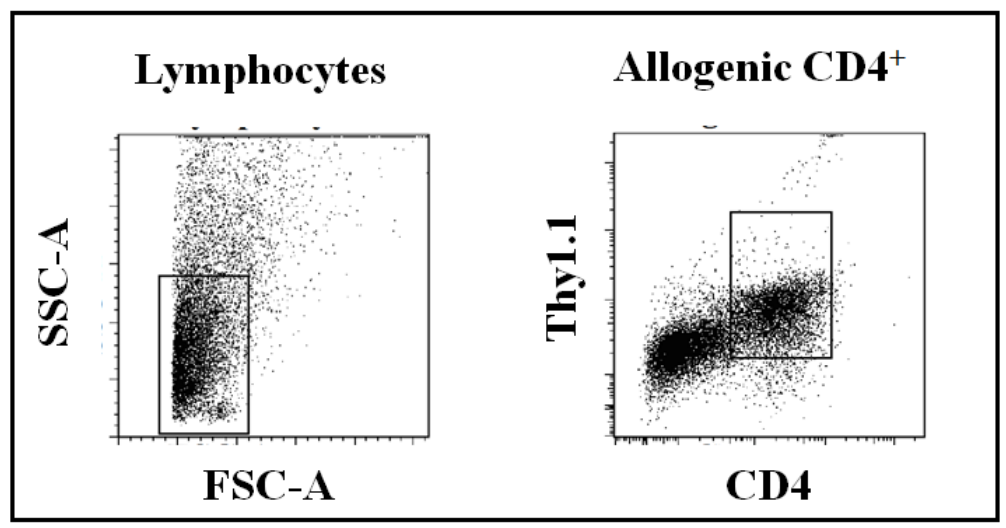

A.7B

Proliferation in Allogenic System

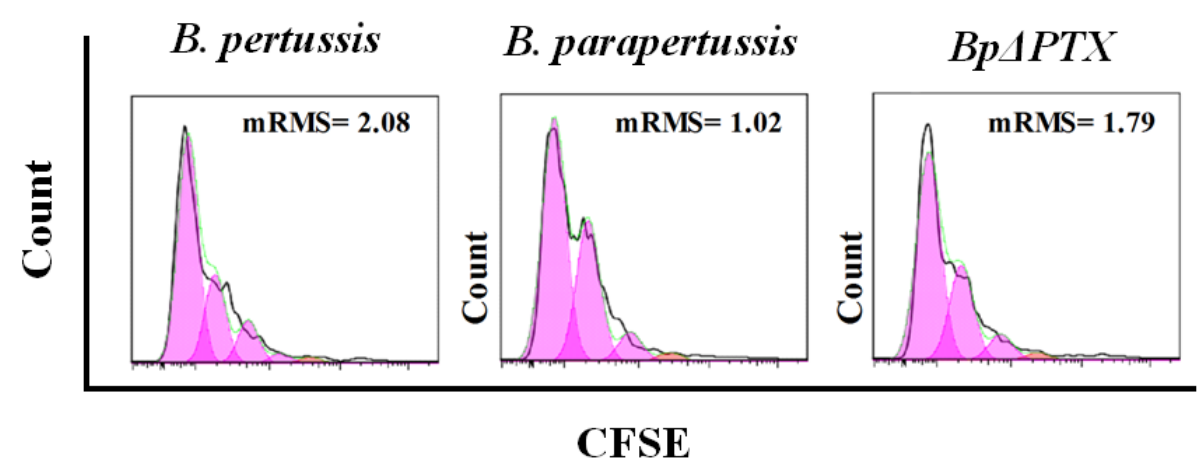

FIG. A.7. Proliferation of the Thy $1.1^{+} / \mathrm{CD4}^{+}$cells in allogeneic system. (A) Lung DCs from Bordetella infections were used to stimulate purified naïve Thy1.1+ CD4+ splenocytes labeled with CFSE (as detailed in material and methods) and cell were cocultured for four days at 1:5 ratio (B) Histograms show CFSE dilution profiles. Approximately 4 cell cycles of division are observed in each system as estimated by FlowJo proliferation analysis. Low mRMS values obtained in every allogeneic system confirm the accuracy of the analysis. 


\section{REFERENCES}

1. Abitorabi, M. A., C. R. Mackay, E. H. Jerome, O. Osorio, E. C. Butcher, and D. J. Erle. 1996. Differential expression of homing molecules on recirculating lymphocytes from sheep gut, peripheral, and lung lymph. J Immunol 156:31113117.

2. Abramson, T., H. Kedem, and D. A. Relman. 2008. Modulation of the NFkappaB pathway by Bordetella pertussis filamentous hemagglutinin. PLoS ONE 3:e3825.

3. Andreasen, C., and N. H. Carbonetti. 2008. Pertussis toxin inhibits early chemokine production to delay neutrophil recruitment in response to Bordetella pertussis respiratory tract infection in mice. Infect Immun 76:5139-5148.

4. Andreasen, C., and N. H. Carbonetti. 2009. Role of neutrophils in response to Bordetella pertussis infection in mice. Infect Immun 77:1182-1188.

5. Andreasen, C., D. A. Powell, and N. H. Carbonetti. 2009. Pertussis toxin stimulates IL-17 production in response to Bordetella pertussis infection in mice. PLoS One 4:e7079.

6. Arico, B., R. Gross, J. Smida, and R. Rappuoli. 1987. Evolutionary relationships in the genus Bordetella. Mol Microbiol 1:301-308.

7. Arico, B., and R. Rappuoli. 1987. Bordetella parapertussis and Bordetella bronchiseptica contain transcriptionally silent pertussis toxin genes. J Bacteriol 169:2847-2853.

8. Avitsur, R., J. L. Stark, F. S. Dhabhar, and J. F. Sheridan. 2002. Social stress alters splenocyte phenotype and function. J Neuroimmunol 132:66-71.

9. Ayala, V. I., J. R. Teijaro, D. L. Farber, S. G. Dorsey, and N. H. Carbonetti. 2011. Bordetella pertussis infection exacerbates influenza virus infection through pertussis toxin-mediated suppression of innate immunity. PLoS One 6:e19016.

10. Bleul, C. C., R. C. Fuhlbrigge, J. M. Casasnovas, A. Aiuti, and T. A. Springer. 1996. A highly efficacious lymphocyte chemoattractant, stromal cellderived factor 1 (SDF-1). J Exp Med 184:1101-1109.

11. Bonato, V. L., A. I. Medeiros, V. M. Lima, A. R. Dias, L. H. Faccioliti, and C. L. Silva. 2001. Downmodulation of CD18 and CD86 on macrophages and VLA-4 on lymphocytes in experimental tuberculosis. Scand J Immunol 54:564-573. 
12. Boursaux-Eude, C., S. Thiberge, G. Carletti, and N. Guiso. 1999. Intranasal murine model of Bordetella pertussis infection: II. Sequence variation and protection induced by a tricomponent acellular vaccine. Vaccine 17:2651-2660.

13. Budde, M. L., J. J. Lhost, D. M. Dudley, E. G. Rakasz, and D. H. O'Connor. Integrin alpha4beta7 is downregulated on the surfaces of simian immunodeficiency virus SIVmac239-infected cells. J Virol 84:6344-6351.

14. Butcher, E. C., and L. J. Picker. 1996. Lymphocyte homing and homeostasis. Science 272:60-66.

15. Campbell, J. J., C. E. Brightling, F. A. Symon, S. Qin, K. E. Murphy, M. Hodge, D. P. Andrew, L. Wu, E. C. Butcher, and A. J. Wardlaw. 2001. Expression of chemokine receptors by lung $\mathrm{T}$ cells from normal and asthmatic subjects. J Immunol 166:2842-2848.

16. Carbonetti, N. H., G. V. Artamonova, C. Andreasen, E. Dudley, R. M. Mays, and Z. E. Worthington. 2004. Suppression of serum antibody responses by pertussis toxin after respiratory tract colonization by Bordetella pertussis and identification of an immunodominant lipoprotein. Infect Immun 72:3350-3358.

17. Clark, J. G., J. B. Mandac-Dy, A. E. Dixon, D. K. Madtes, K. M. Burkhart, J. M. Harlan, and D. C. Bullard. 2004. Trafficking of Th1 cells to lung: a role for selectins and a P-selectin glycoprotein-1-independent ligand. Am J Respir Cell Mol Biol 30:220-227.

18. de Bree, G. J., J. Heidema, E. M. van Leeuwen, G. M. van Bleek, R. E. Jonkers, H. M. Jansen, R. A. van Lier, and T. A. Out. 2005. Respiratory syncytial virus-specific CD8+ memory $\mathrm{T}$ cell responses in elderly persons. J Infect Dis 191:1710-1718.

19. de Bree, G. J., E. M. van Leeuwen, T. A. Out, H. M. Jansen, R. E. Jonkers, and R. A. van Lier. 2005. Selective accumulation of differentiated CD8+ T cells specific for respiratory viruses in the human lung. J Exp Med 202:1433-1442.

20. Debes, G. F., C. N. Arnold, A. J. Young, S. Krautwald, M. Lipp, J. B. Hay, and E. C. Butcher. 2005. Chemokine receptor CCR7 required for T lymphocyte exit from peripheral tissues. Nat Immunol 6:889-894.

21. Fan, H., O. M. Peck, G. E. Tempel, P. V. Halushka, and J. A. Cook. 2004. Toll-like receptor 4 coupled GI protein signaling pathways regulate extracellular signal-regulated kinase phosphorylation and AP-1 activation independent of NFkappaB activation. Shock 22:57-62. 
22. Fedele, G., M. Nasso, F. Spensieri, R. Palazzo, L. Frasca, M. Watanabe, and C. M. Ausiello. 2008. Lipopolysaccharides from Bordetella pertussis and Bordetella parapertussis differently modulate human dendritic cell functions resulting in divergent prevalence of Th17-polarized responses. J Immunol 181:208-216.

23. Fedele, G., F. Spensieri, R. Palazzo, M. Nasso, G. Y. Cheung, J. G. Coote, and C. M. Ausiello. Bordetella pertussis commits human dendritic cells to promote a Th1/Th17 response through the activity of adenylate cyclase toxin and MAPK-pathways. PLoS One 5:e8734.

24. Gameiro, J., P. R. Nagib, C. F. Andrade, D. M. Villa-Verde, S. D. SilvaBarbosa, W. Savino, F. T. Costa, and L. Verinaud. Changes in cell migrationrelated molecules expressed by thymic microenvironment during experimental Plasmodium berghei infection: consequences on thymocyte development. Immunology 129:248-256.

25. Garcia, A., J. Niubo, M. A. Benitez, M. Viqueira, and J. L. Perez. 1996. Comparison of two leukocyte extraction methods for cytomegalovirus antigenemia assay. J Clin Microbiol 34:182-184.

26. Guermonprez, P., N. Khelef, E. Blouin, P. Rieu, P. Ricciardi-Castagnoli, N. Guiso, D. Ladant, and C. Leclerc. 2001. The adenylate cyclase toxin of Bordetella pertussis binds to target cells via the alpha(M)beta(2) integrin (CD11b/CD18). J Exp Med 193:1035-1044.

27. Hauck, C. R., F. Agerer, P. Muenzner, and T. Schmitter. 2006. Cellular adhesion molecules as targets for bacterial infection. Eur J Cell Biol 85:235-242.

28. Hodge, G., S. Hodge, C. Markus, A. Lawrence, and P. Han. 2003. A marked decrease in L-selectin expression by leucocytes in infants with Bordetella pertussis infection: leucocytosis explained? Respirology 8:157-162.

29. Holt, P. G., D. H. Strickland, M. E. Wikstrom, and F. L. Jahnsen. 2008. Regulation of immunological homeostasis in the respiratory tract. Nat Rev Immunol 8:142-152.

30. Hudnall, S. D., and C. P. Molina. 2000. Marked increase in L-selectin-negative $\mathrm{T}$ cells in neonatal pertussis. The lymphocytosis explained? Am J Clin Pathol 114:35-40.

31. Huh, J. C., D. H. Strickland, F. L. Jahnsen, D. J. Turner, J. A. Thomas, S. Napoli, I. Tobagus, P. A. Stumbles, P. D. Sly, and P. G. Holt. 2003.

Bidirectional interactions between antigen-bearing respiratory tract dendritic cells 
(DCs) and T cells precede the late phase reaction in experimental asthma: DC activation occurs in the airway mucosa but not in the lung parenchyma. J Exp Med 198:19-30.

32. Kabashima, K., K. Sugita, N. Shiraishi, H. Tamamura, N. Fujii, and Y. Tokura. 2007. CXCR4 engagement promotes dendritic cell survival and maturation. Biochem Biophys Res Commun 361:1012-1016.

33. Kamath, A. T., C. E. Sheasby, and D. F. Tough. 2005. Dendritic cells and NK cells stimulate bystander T cell activation in response to TLR agonists through secretion of IFN-alpha beta and IFN-gamma. J Immunol 174:767-776.

34. Kang, S. G., J. Park, J. Y. Cho, B. Ulrich, and C. H. Kim. Complementary roles of retinoic acid and TGF-beta1 in coordinated expression of mucosal integrins by T cells. Mucosal Immunol 4:66-82.

35. Lentschat, A., H. Karahashi, K. S. Michelsen, L. S. Thomas, W. Zhang, S. N. Vogel, and M. Arditi. 2005. Mastoparan, a G protein agonist peptide, differentially modulates TLR4- and TLR2-mediated signaling in human endothelial cells and murine macrophages. J Immunol 174:4252-4261.

36. Luster, A. D., R. Alon, and U. H. von Andrian. 2005. Immune cell migration in inflammation: present and future therapeutic targets. Nat Immunol 6:1182-1190.

37. Lyons, A. B., and K. V. Doherty. 2004. Flow cytometric analysis of cell division by dye dilution. Curr Protoc Cytom Chapter 9:Unit 911.

38. Martin, C., K. B. Uribe, G. Gomez-Bilbao, and H. Ostolaza. 2011. Adenylate cyclase toxin promotes internalisation of integrins and raft components and decreases macrophage adhesion capacity. PLoS One 6:e17383.

39. Mattoo, S., and J. D. Cherry. 2005. Molecular pathogenesis, epidemiology, and clinical manifestations of respiratory infections due to Bordetella pertussis and other Bordetella subspecies. Clin Microbiol Rev 18:326-382.

40. McIntyre, P., and N. Wood. 2009. Pertussis in early infancy: disease burden and preventive strategies. Curr Opin Infect Dis 22:215-223.

41. Menezes, G. B., W. Y. Lee, H. Zhou, C. C. Waterhouse, D. C. Cara, and P. Kubes. 2009. Selective down-regulation of neutrophil Mac-1 in endotoxemic hepatic microcirculation via IL-10. J Immunol 183:7557-7568.

42. Morimoto, C., S. Iwata, and K. Tachibana. 1998. VLA-4-mediated signaling. Curr Top Microbiol Immunol 231:1-22. 
43. Munoz, J. J., H. Arai, R. K. Bergman, and P. L. Sadowski. 1981. Biological activities of crystalline pertussigen from Bordetella pertussis. Infect Immun 33:820-826.

44. Oka, T., T. Honda, K. Morokuma, A. Ginnaga, K. Ohkuma, and M. Sakoh. 1994. Enhancing effects of pertussis toxin B oligomer on the immunogenicity of influenza vaccine administered intranasally. Vaccine 12:1255-1258.

45. Pero, R. S., M. T. Borchers, K. Spicher, S. I. Ochkur, L. Sikora, S. P. Rao, H. Abdala-Valencia, K. R. O'Neill, H. Shen, M. P. McGarry, N. A. Lee, J. M. Cook-Mills, P. Sriramarao, M. I. Simon, L. Birnbaumer, and J. J. Lee. 2007. Galphai2-mediated signaling events in the endothelium are involved in controlling leukocyte extravasation. Proc Natl Acad Sci U S A 104:4371-4376.

46. Pham, T. H., T. Okada, M. Matloubian, C. G. Lo, and J. G. Cyster. 2008. $\mathrm{S} 1 \mathrm{P} 1$ receptor signaling overrides retention mediated by $\mathrm{G}$ alpha i-coupled receptors to promote $\mathrm{T}$ cell egress. Immunity 28:122-133.

47. Picker, L. J., R. J. Martin, A. Trumble, L. S. Newman, P. A. Collins, P. R. Bergstresser, and D. Y. Leung. 1994. Differential expression of lymphocyte homing receptors by human memory/effector T cells in pulmonary versus cutaneous immune effector sites. Eur J Immunol 24:1269-1277.

48. Picker, L. J., S. A. Michie, L. S. Rott, and E. C. Butcher. 1990. A unique phenotype of skin-associated lymphocytes in humans. Preferential expression of the HECA-452 epitope by benign and malignant T cells at cutaneous sites. Am J Pathol 136:1053-1068.

49. Pittman, M., B. L. Furman, and A. C. Wardlaw. 1980. Bordetella pertussis respiratory tract infection in the mouse: pathophysiological responses. J Infect Dis 142:56-66.

50. Pribila, J. T., A. C. Quale, K. L. Mueller, and Y. Shimizu. 2004. Integrins and T cell-mediated immunity. Annu Rev Immunol 22:157-180.

51. Ray, S. J., S. N. Franki, R. H. Pierce, S. Dimitrova, V. Koteliansky, A. G. Sprague, P. C. Doherty, A. R. de Fougerolles, and D. J. Topham. 2004. The collagen binding alpha1beta1 integrin VLA-1 regulates CD8 T cell-mediated immune protection against heterologous influenza infection. Immunity 20:167179.

52. Ross, P. J., E. C. Lavelle, K. H. Mills, and A. P. Boyd. 2004. Adenylate cyclase toxin from Bordetella pertussis synergizes with lipopolysaccharide to promote 
innate interleukin-10 production and enhances the induction of Th2 and regulatory T cells. Infect Immun 72:1568-1579.

53. Sallusto, F., C. R. Mackay, and A. Lanzavecchia. 2000. The role of chemokine receptors in primary, effector, and memory immune responses. Annu Rev Immunol 18:593-620.

54. Schneider, O. D., A. A. Weiss, and W. E. Miller. 2009. Pertussis toxin signals through the TCR to initiate cross-desensitization of the chemokine receptor CXCR4. J Immunol 182:5730-5739.

55. Shen, S., M. I. Chuck, M. Zhu, D. M. Fuller, C. W. Yang, and W. Zhang. 2010. The importance of LAT in the activation, homeostasis, and regulatory function of T cells. J Biol Chem 285:35393-35405.

56. Sigmundsdottir, H., and E. C. Butcher. 2008. Environmental cues, dendritic cells and the programming of tissue-selective lymphocyte trafficking. Nat Immunol 9:981-987.

57. Solovjov, D. A., E. Pluskota, and E. F. Plow. 2005. Distinct roles for the alpha and beta subunits in the functions of integrin alphaMbeta2. J Biol Chem 280:1336-1345.

58. Sozzani, S., P. Allavena, G. D'Amico, W. Luini, G. Bianchi, M. Kataura, T. Imai, O. Yoshie, R. Bonecchi, and A. Mantovani. 1998. Differential regulation of chemokine receptors during dendritic cell maturation: a model for their trafficking properties. J Immunol 161:1083-1086.

59. Stewart, P. L., and G. R. Nemerow. 2007. Cell integrins: commonly used receptors for diverse viral pathogens. Trends Microbiol 15:500-507.

60. Thatte, J., V. Dabak, M. B. Williams, T. J. Braciale, and K. Ley. 2003. LFA-1 is required for retention of effector CD8 T cells in mouse lungs. Blood 101:49164922.

61. Verma, A., A. M. Cheung, and D. L. Burns. 2008. Stabilization of the pertussis toxin secretion apparatus by the C terminus of PtlD. J Bacteriol 190:7285-7290.

62. Weiss, A. A., E. L. Hewlett, G. A. Myers, and S. Falkow. 1983. Tn5-induced mutations affecting virulence factors of Bordetella pertussis. Infect Immun 42:3341.

63. Wiedle, G., D. Dunon, and B. A. Imhof. 2001. Current concepts in lymphocyte homing and recirculation. Crit Rev Clin Lab Sci 38:1-31. 
64. Woodland, D. L., and J. E. Kohlmeier. 2009. Migration, maintenance and recall of memory T cells in peripheral tissues. Nat Rev Immunol 9:153-161.

65. Worthington, Z. E., N. Van Rooijen, and N. H. Carbonetti. 2011. Enhancement of Bordetella parapertussis infection by Bordetella pertussis in mixed infection of the respiratory tract. FEMS Immunol Med Microbiol 63:119128.

66. Zabel, B. A., W. W. Agace, J. J. Campbell, H. M. Heath, D. Parent, A. I. Roberts, E. C. Ebert, N. Kassam, S. Qin, M. Zovko, G. J. LaRosa, L. L. Yang, D. Soler, E. C. Butcher, P. D. Ponath, C. M. Parker, and D. P. Andrew. 1999. Human G protein-coupled receptor GPR-9-6/CC chemokine receptor 9 is selectively expressed on intestinal homing $\mathrm{T}$ lymphocytes, mucosal lymphocytes, and thymocytes and is required for thymus-expressed chemokine-mediated chemotaxis. J Exp Med 190:1241-1256. 